\title{
Finite-fault source inversion using adjoint methods in 3-D heterogeneous media
}

\author{
Surendra Nadh Somala, ${ }^{1}$ Jean-Paul Ampuero ${ }^{2}$ and Nadia Lapusta ${ }^{3}$ \\ ${ }^{1}$ Department of Civil Engineering, Indian Institute of Technology Hyderabad, Telangana 502285, India. E-mail: somalasurendranadh@gmail.com \\ ${ }^{2}$ Seismological Laboratory, California Institute of Technology, Pasadena, CA 91125, USA \\ ${ }^{3}$ Seismological Laboratory and Department of Mechanical and Civil Engineering, California Institute of Technology, Pasadena, CA 91125, USA
}

Accepted 2018 April 11. Received 2017 November 22; in original form 2018 April 4

\begin{abstract}
SUMMAR Y
Accounting for lateral heterogeneities in the 3-D velocity structure of the crust is known to improve earthquake source inversion, compared to results based on 1-D velocity models which are routinely assumed to derive finite-fault slip models. The conventional approach to include known 3-D heterogeneity in source inversion involves pre-computing 3-D Green's functions, which requires a number of 3-D wave propagation simulations proportional to the number of stations or to the number of fault cells. The computational cost of such an approach is prohibitive for the dense data sets that could be provided by future earthquake observation systems. Here, we propose an adjoint-based optimization technique to invert for the spatiotemporal evolution of slip velocity. The approach does not require pre-computed Green's functions. The adjoint method provides the gradient of the cost function, which is used to improve the model iteratively employing an iterative gradient-based minimization method. The adjoint approach is shown to be computationally more efficient than the conventional approach based on pre-computed Green's functions in a broad range of situations. We consider data up to $1 \mathrm{~Hz}$ from a Haskell source scenario (a steady pulse-like rupture) on a vertical strikeslip fault embedded in an elastic 3-D heterogeneous velocity model. The velocity model comprises a uniform background and a 3-D stochastic perturbation with the von Karman correlation function. Source inversions based on the 3-D velocity model are performed for two different station configurations, a dense and a sparse network with 1 and $20 \mathrm{~km}$ station spacing, respectively. These reference inversions show that our inversion scheme adequately retrieves the rise time when the velocity model is exactly known, and illustrates how dense coverage improves the inference of peak-slip velocities. We investigate the effects of uncertainties in the velocity model by performing source inversions based on an incorrect, homogeneous velocity model. We find that, for velocity uncertainties that have standard deviation and correlation length typical of available 3-D crustal models, the inverted sources can be severely contaminated by spurious features even if the station density is high. When data from thousand or more receivers is used in source inversions in 3-D heterogeneous media, the computational cost of the method proposed in this work is at least two orders of magnitude lower than source inversion based on pre-computed Green's functions.
\end{abstract}

Key words: Inverse theory; Numerical modelling; 3D Waveform inversion; Computational seismology; 3D Wave propagation.

\section{INTRODUCTION}

Earthquake source inversion is the process of inferring the spatiotemporal distribution of slip rate on an assumed fault surface from a combination of seismological, geodetic, remote sensing, tsunami and field observations (Ide et al. 2005). The inferred source parameters inform fundamental studies in earthquake physics and applied studies in earthquake engineering. Valuable constraints on earthquake rupture processes can in principle be derived from groundmotion recordings. In particular, seismological data provide a window into the time-dependency of the rupture process.

The reliability of earthquake source inversion can depend, among other factors, on the quality of the assumed velocity model, that is, the spatial distribution of seismic wave velocities in the medium. Earthquake source inversion is typically performed with Green's 
functions computed under the assumption of a horizontally layered crust. Lateral heterogeneity is accounted for only approximately, for instance through station correction factors (Shao et al. 2012) or through station-specific layered models (Liu et al. 2006; Asano \& Iwata 2009). Graves \& Wald (2001) showed that incorporating well-calibrated 3-D Green's functions provides a better reconstruction of the source. The development of 3-D crustal velocity models is in steady progress. In southern California, wave propagation simulations based on the SCEC CVM-H model are consistent with observed waveforms down to periods of a few seconds (Tape et al. 2009). However, for dense seismic networks and fine resolution source parametrizations, the computation and storage of 3-D Green's functions can be prohibitively demanding, and an alternative approach for source inversion is desirable.

Inversions for earthquakes with few recordings are found to be inconsistent across source inversion modelling groups (Beresnev 2003; Mai et al. 2016). An example of poorly recorded earthquake where different groups produced widely different source models is the 1999 Izmit earthquake in Turkey. Beresnev (2003) found little resemblance among five published source models of this earthquake (Bouchon et al. 2002; Delouis et al. 2002; Gülen et al. 2002; Li et al. 2002; Sekiguchi \& Iwata 2002) in terms of asperity locations and slip distribution. The 1999 Chi-Chi earthquake in Taiwan was recorded by several hundreds of stations (Lee et al. 2001). Ide et al. (2005) compared four published source models of this event (Chi et al. 2001; Ma et al. 2001; Yu et al. 2001; Zeng \& Chen 2001) and found that they are very similar in terms of rupture length, width, duration and propagation velocity. Despite the differences in the assumed velocity structure and modelling approaches, the dense distribution of the Chi-Chi earthquake data set enabled robust constraints on the source parameters.

Densifying seismic networks is an important path towards more reliable source imaging. Denser coverage allows capturing better the spatio-temporal complexity of the Green's functions. Block-byblock networks of low-cost sensors (Clayton et al. 2012) could soon provide ground-motion recordings every few hundreds of meters in urban areas. Emergent concepts for space-based earthquake observation systems (Michel et al. 2013) could expand such dense coverage to remote areas. A systematic assessment of the effect of network density on the quality of the source recovery requires a scalable source inversion approach that can handle efficiently very large numbers of stations.

Additional constraints are typically imposed on source inversions, including total seismic moment, minimum norm, minimum roughness, positivity or bounds on rupture speed and rise time. Some of these constraints have a strong physical basis. For instance, constraining the rupture speed to be slower than the $P$ wave speed naturally enforces the causality principle if the hypocentre location is assumed. In contrast, constraints on the rise time are usually motivated by a desire to reduce the number of unknowns in order to mitigate the non-uniqueness of the inverse problem, rather than by known physical bounds on the rise time. Dynamic rupture simulations can produce a wide variety of behaviours depending on initial conditions and friction parameters. One example of such complexity is the occurrence of rupture reversals, that is, rupture propagation in the direction opposite to that of the ongoing rupture, as was suggested for the 2010 El Mayor-Cucapah earthquake (http://www.scec.org/meetings/2011am/emc.html) and for the 1984 Morgan Hill earthquake (Beroza \& Spudich 1988). Another example is the occurrence of multiple pulses (repeated slip) during the 2011 Tohoku earthquake inferred by Lee et al. (2011) and discussed through dynamic rupture models by Gabriel et al. (2012) and Galvez et al. (2016). Capturing such complex rupture processes requires a flexible parametrization with minimal constraints.

Here, we address the following question: How does one efficiently perform seismic source inversion based on dense seismological data sets and minimal prior assumptions on the source while taking into account the lateral heterogeneity of the medium? To address this, we introduce a scalable adjoint-based method for finite source inversion that accounts for known 3-D heterogeneities of the crustal velocity model without pre-computed Green's functions.

Adjoint methods (Tarantola 1984) have been in use in seismology for tomography (Tromp et al. 2005; Askan \& Bielak 2008; Liu \& Tromp 2008; Fichtner et al. 2010; Tape et al. 2010) and point-source moment-tensor inversion (Hjörleifsdóttir et al. 2007; Kim et al. 2011). The adjoint fields are related to time-reversal wave propagation modelling, and time-reversal source imaging can be seen as the first iteration in a source inversion problem solved by an iterative method (Kawakatsu \& Montagner 2008). Attempts to achieve finitefault source imaging through time-reversal have been unsuccessful in resolving rupture details (Kremers et al. 2011). Here, we present a method for source inversion based on adjoint methods, iteratively updating the source model through time-reversal simulations. We show that the gradient of the cost function with respect to slip rate is given by the tractions on the fault plane in the adjoint problem. This is different from the moment-tensor (density) source inversion (Kremers et al. 2011; Hjörleifsdóttir et al. 2007) for which the adjoint fields are strains. Our formulation is related to that of Gallovič et al. (2009), although they did not provide an interpretation of the adjoint field and they used pre-computed response functions which, as we will show, are suitable for situations with few source parameters and few stations.

The remainder of this paper is organized as follows. In Section 2, we introduce a method for finite source inversion that accounts for a 3-D velocity model and does not require pre-computed Green's functions. In Section 3, we compare its computational cost to the prevailing approach based on pre-computed Green's functions. In Section 4, we apply our method to simulated scenarios that illustrate the impact of insufficient knowledge of the velocity model on finite source inversion. The method and results are further discussed in Section 5. Section 6 summarizes our findings.

\section{THEORY AND METHODOLOGY}

\subsection{Problem statement}

We aim at inferring the spatio-temporal distribution of slip velocity on an assumed fault surface from ground-motion data recorded at (or near) the Earth's surface. We focus here on seismological data, the primary data set to constrain the time-dependency of the rupture process. Other data sets like GPS or teleseismic waveforms could be included in our source inversion formulation, at the expense of additional complexity in determining the optimal weighting for the different data sets (Sekiguchi et al. 2000; Ide et al. 2005).

The data comprise three-component ground velocity time-series $\dot{\mathbf{d}}\left(\mathbf{x}_{\mathbf{r}}, t\right)$ recorded at a set of $N_{\text {sta }}$ receiver locations $\mathbf{x}_{\mathbf{r}}$ between the initiation of rupture at $t=0$ and the final recording time $t=T$. The model comprises the two-component slip velocity time-series $\mathbf{m}(\mathbf{x}, t)$ at all points $\mathbf{x}$ on the assumed fault surface $\Sigma$ (we leave the rake unconstrained). We use the term 'synthetics' and the notation $\dot{\mathbf{s}}\left(\mathbf{x}_{\mathbf{r}}, t, \mathbf{m}\right)$ to denote ground velocities computed at the receiver location $\mathbf{x}_{\mathbf{r}}$ based on the source model $\mathbf{m}$. The synthetic time-series and model parameters are linearly related by a partial differential 
equation, the seismic wave equation, or equivalently by a representation theorem (e.g. section 3.2 of Aki \& Richards 2002). We concisely write this relation as

$\dot{\mathbf{s}}=\mathbf{G} \mathbf{m}$,

where $\mathbf{G}$ is a linear operator from the model space to the data space. We seek a model that reproduces the observed wavefield, $\dot{\mathbf{s}}(\mathbf{m}) \approx \dot{\mathbf{d}}$, in a sense that will be made precise now.

Waveform data are usually low-pass filtered prior to earthquake source inversion in order to down weight those high-frequency components of the wavefield that cannot be well predicted due to the coarseness of the available crustal velocity models. Depending on the sensor type, high-pass filter is often applied too, to down weight frequencies with low signal-to-noise level. We denote by $h(t)$ the impulse time response of the filter and by $*$ the convolution operation between two time-series. We define a cost function $\chi$ that quantifies the misfit between the filtered data and synthetics:

$\chi(\mathbf{m})=\frac{1}{2} \int_{0}^{T} \sum_{r=1}^{N_{\text {sta }}} W_{r}\left\|h(t) *\left(\dot{\mathbf{s}}\left(\mathbf{x}_{\mathbf{r}}, t, \mathbf{m}\right)-\dot{\mathbf{d}}\left(\mathbf{x}_{\mathbf{r}}, t\right)\right)\right\|^{2} \mathrm{~d} t$,

where $W_{r}$ is a data weight for the receiver located at $\mathbf{x}_{\mathbf{r}}$ and $\|\cdot\|$ is the 3-D Euclidian norm. Our goal is to find the source model $\mathbf{m}$ that minimizes the cost function $\chi$, subject to eq. (1). The optimal model in this classical least squares problem is the solution of the so-called normal equations (Tarantola 2005)

$\mathbf{G}^{\dagger} \mathbf{F}^{\dagger} \mathbf{W} \mathbf{F}(\mathbf{G} \mathbf{m}-\mathbf{d})=0$,

where $\mathbf{W}$ is the spatial data-weighting operator, $\mathbf{F}$ the temporal datafilter operator (in frequency domain it is simply a multiplicative weighting operator), $\mathbf{F}^{\dagger}$ is its adjoint, and $\mathbf{G}^{\dagger}$ is the adjoint operator of $\mathbf{G} . \mathbf{G}^{\dagger}$ is defined as the linear operator from the data space to the model space that satisfies the relation (Tarantola 2005)

$\left\langle\mathbf{d}^{\prime}, \mathbf{G} \mathbf{m}^{\prime}\right\rangle=\left\langle\mathbf{G}^{\dagger} \mathbf{d}^{\prime}, \mathbf{m}^{\prime}\right\rangle$

for any arbitrary data $\mathbf{d}^{\prime}$ and model $\mathbf{m}^{\prime}$, where $\langle\cdot, \cdot\rangle$ denotes the natural dot product in the data space on the left-hand side or in the model space on the right-hand side.

Upon discretizating the space and time dimensions, and representing the model $\mathbf{m}$ by a discrete set of space-time basis functions, we obtain the classical linear formulation of the source inversion problem (e.g. Hartzell \& Heaton 1983; Lee 2012) in which the unknowns are the slip rates at each fault cell and on multiple time windows. In standard practice, the slip-velocity unknowns are restricted to time intervals much shorter than the whole rupture duration (Olson \& Anderson 1988; Das \& Suhadolc 1996; Gallovič \& Zahradník 2012). At each fault location, these intervals are dictated by assumed higher bounds on the rupture speed and rise time (Olson \& Anderson 1988; Das \& Suhadolc 1996; Gallovič \& Zahradník 2012). In contrast to standard practice, we consider the values of slip velocity at every instant as unknowns. That is, we do not enforce any prior information on hypocentre location, rupture speed, rise time and total rupture duration, although these may be incorporated in our formulation when warranted. This allows for any fault location to rupture multiple times and for the inversion to capture complex rupture histories that are physically admissible, for example, secondary rupture fronts running in the opposite direction as the main front.

\subsection{Overview of the iterative procedure for source inversion}

A discrete version of eq. (1) is obtained by restricting the model search to the model subspace spanned by a finite set of basis functions. Moreover, the data space is also discretized by temporal sampling of the seismograms. In the conventional approach, $\mathbf{G}$ is a matrix containing Green's functions, that is, discretely sampled seismograms computed in response to excitation of sources given by each slip basis function, computed and stored once before the inversion starts. The matrix $\mathbf{G}$ has as many rows as the dimension of the data space and as many columns as the dimension of the model space. Its general form is given in, for example, Olson \& Apsel (1982). We refer to this approach as the pre-computed Green's function approach, or for short 'the GF approach'. In contrast, the adjoint approach proposed here does not require computing and storing a matrix $\mathbf{G}$, it only requires a procedure to compute the products $\mathbf{G} \mathbf{m}^{\prime}$ and $\mathbf{G}^{\dagger} \mathbf{d}^{\prime}$ for any model $\mathbf{m}^{\prime}$ and data $\mathbf{d}^{\prime}$. $\mathbf{G} \mathbf{m}^{\prime}$ is simply the result of a wave propagation simulation with source $\mathbf{m}^{\prime}$. The procedure to obtain $\mathbf{G}^{\dagger} \mathbf{d}^{\prime}$ will be developed in the next section. In the GF approach, $\mathbf{G}^{\dagger}$ is simply the transpose matrix of $\mathbf{G}$.

We solve the normal eq (3) by applying a standard iterative algorithm, the conjugate gradient (CG) method (Hestenes \& Stiefel 1952; Polak \& Ribière 1969). The algorithm applies to both the GF approach and adjoint approach. A similar CG-based approach to line-source retrieval from regional data was developed by Gallovič et al. (2009) for the 2008 Movri Mountain earthquake. The algorithm is shown pictorially in Fig. 1 and its step-by-step description is as follows:

(1) Initialize the iteration counter, $k=0$. Filter the data, $\mathbf{F} \mathbf{d}$, where $\mathbf{F}$ is a matrix containing filter coefficients.

(2) Assume an initial model, $\mathbf{m}^{0}$, and compute the corresponding synthetics, $\mathbf{s}^{0}=\mathbf{G ~ m}^{0}$.

(3) Compute residuals by subtracting the synthetics from the data, $\mathbf{r}^{0}=\mathbf{F} \mathbf{s}^{0}-\mathbf{F} \mathbf{d}$.

(4) Compute the gradient of the cost function with respect to the model parameters, $\boldsymbol{\gamma}^{0}=\mathbf{G}^{\dagger} \mathbf{F}^{\dagger} \mathbf{r}^{0}$.

(5) Set the search direction, $\mathbf{p}^{0}=-\boldsymbol{\gamma}^{0}$.

(6) Compute new synthetics, $\mathbf{s}^{k}=\mathbf{G} \mathbf{p}^{k}$.

(7) Update the model so that the cost function is minimized along the search direction, $\mathbf{m}^{k+1}=\mathbf{m}^{k}+\alpha \mathbf{p}^{k}$, where $\alpha=$ $\left\langle\mathbf{r}^{k}, \mathbf{F} \mathbf{s}^{k}\right\rangle /\left\langle\mathbf{F} \mathbf{s}^{k}, \mathbf{F} \mathbf{s}^{k}\right\rangle$.

(8) Update the residuals, $\mathbf{r}^{k+1}=\mathbf{r}^{k}+\alpha \mathbf{F} \mathbf{s}^{k}$.

(9) Compute the new gradient, $\boldsymbol{\gamma}^{k+1}=\mathbf{G}^{\dagger} \mathbf{F}^{\dagger} \mathbf{r}^{k+1}$.

(10) Update the search direction applying the PolakRibiere formula, $\mathbf{p}^{k+1}=-\boldsymbol{\gamma}^{k+1}+\beta \mathbf{p}^{k}$, where $\beta=\left\langle\boldsymbol{\gamma}^{k+1}-\right.$ $\left.\boldsymbol{\gamma}^{k}, \boldsymbol{\gamma}^{k+1}\right\rangle /\left\langle\boldsymbol{\gamma}^{k}, \boldsymbol{\gamma}^{k}\right\rangle$.

(11) If the norm of the new search direction $\mathbf{p}^{k+1}$ is less than a prescribed tolerance, stop. Otherwise, increment the iteration counter, $k \rightarrow k+1$, and go to step 6 .

The particular solution to which the CG algorithm converges depends on the choice of the initial guess. Here we set $\mathbf{m}^{0}=0$. With this choice, the CG algorithm converges to the minimum-norm solution. This choice is not motivated by physical considerations on the rupture process, but rather by the insight it provides on the intrinsic uncertainties of the source inversion problem. The inverse problem of earthquake source retrieval from ground motions recorded over a limited region of the Earth's surface and with a limited frequency band generally suffers from non-uniqueness. The minimum-norm solution is orthogonal to the null space of the inverse problem, 


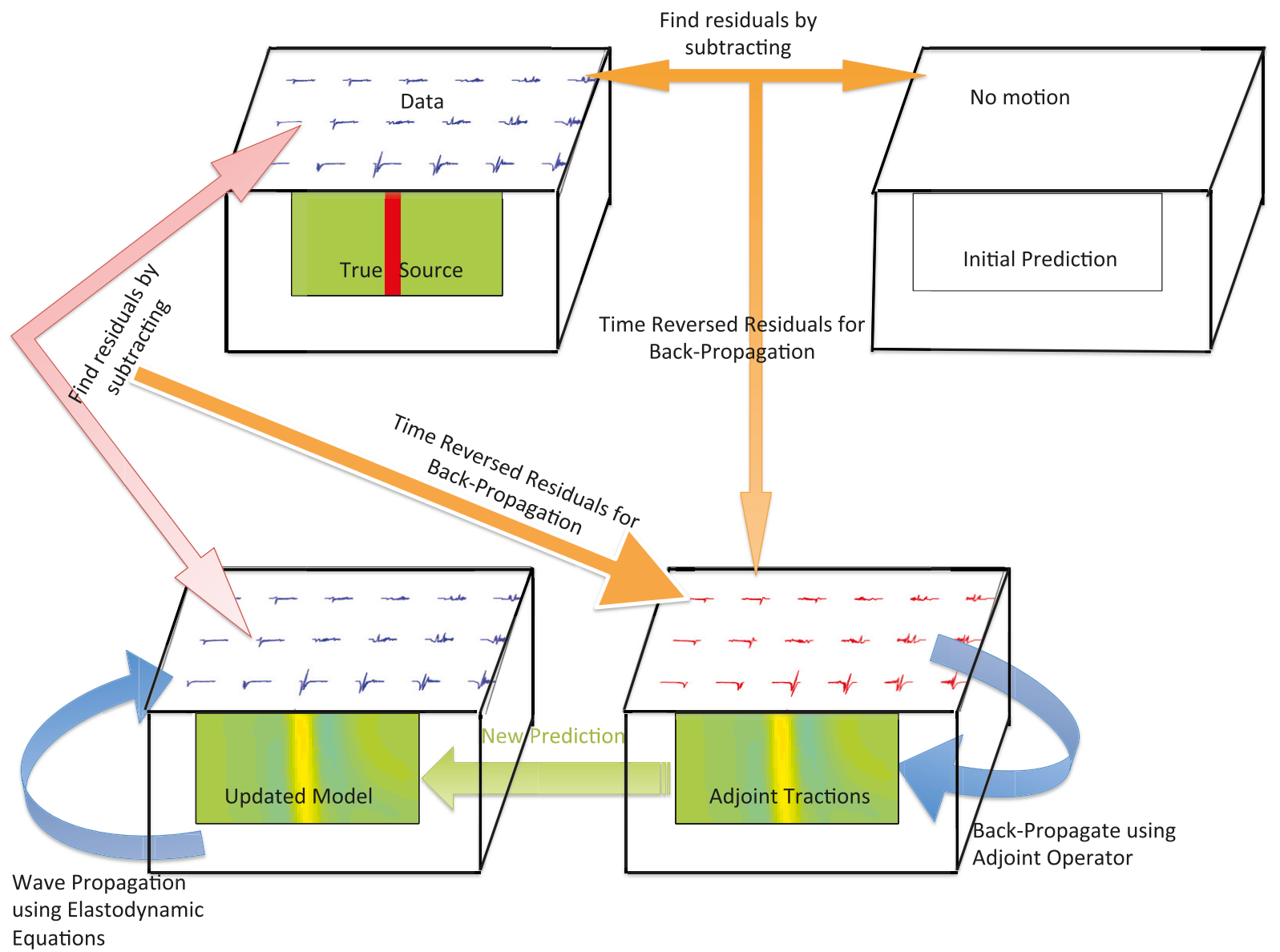

Figure 1. Sketch of the adjoint method illustrating the iterative procedure to update the source model using forward and backward (adjoint) wave propagation simulations. If only the first adjoint simulation is carried out, then the procedure is equivalent to time-reversal source imaging. Each back propagation retrieves the gradient of the misfit function represented by tractions recorded on the fault. The forward wave propagation at each iteration other than the initial one uses the current model and gives the synthetics of the current iteration.

hence it isolates the part of the earthquake rupture that is "visible' by a given data set. The relation between the visible model space and the complete model space may be complicated enough that physical interpretation of the minimum-norm solution is not straightforward. For this reason, caution should be exercised to not overly interpret the minimum-norm solution. Gallovič \& Zahradník (2011) gave an example of a spurious asperity in a minimum-norm solution, a feature in the visible portions of the source that seems physically plausible but is not real. The real source is made of its visible part plus other 'invisible' parts that lie in the null space of the inverse problem. Enforcing additional constraints in the source inversion allows to populate the invisible components of the source, but whether this approach takes us closer to the true source depends on the degree of certainty of the constraints. In particular, if the constraints are artificial (regularization) or derived from uncertain or restrictive physical considerations, they can bias source recovery despite reducing model covariance.

In the adjoint approach, steps 2 and 6 involve a wave propagation simulation with prescribed kinematic source. In the next section, we show that steps 4 and 9 are accomplished by a wave propagation simulation with the time-reversed ground-motion residuals applied as point forces at the receiver locations. Hence, each CG iteration in the adjoint approach requires one forward-propagation simulation (during the model update, step 6) and one back-propagation simulation (during the gradient update, step 9). In contrast, the GF approach involves temporal convolution with the Green's functions and spatial summation over all subfaults in step 6 , and cross-correlation with the Green's functions and spatial summation over all stations at step 9 .

For the source inversion with the GF approach using the CG algorithm, Somala et al. (2014) found, by comparing the inverted models at various iterations, that convergence is obtained in about 250 iterations when the velocity model is perfectly known. In practice, the velocity model has uncertainties and continuing the CG iterations will eventually map structural uncertainties into spurious features of the source model. The number of CG iterations, $N_{\text {iter }}$, plays the role of regularization because the features of the source that are less robust to uncertainties in the data and in the velocity model are associated with smaller eigenvalues of $\mathbf{G}^{\dagger} \mathbf{G}$ and converge slower. Although a general-purpose iteration arrest criterion for CG is not available, the number of iterations in practice should certainly be significantly smaller than in the ideal case considered by Somala et al. (2014), $N_{\text {iter }} \ll 250$. 


\subsection{Lagrangian formulation of the adjoint problem}

Here we compute the gradient of the cost function with respect to the source model by solving an adjoint problem. We use a Lagrangian formulation modified from that presented by Liu \& Tromp (2006) and pose it as a boundary control problem. We formulate the Lagrangian based on filtered and weighted residuals. Simulations are done on a bounded domain $\Omega$, the boundary of which comprises a free surface $\partial \Omega_{1}$ and an absorbing boundary $\partial \Omega_{2}$. We denote by $\rho$ the density and $\mathbf{C}$ the fourth-order elastic stiffness tensor of the crust. These material properties can be spatially non uniform. The Lagrangian is given by

$$
\begin{aligned}
\mathcal{L}(\dot{\mathbf{s}}, \mathbf{m}, \boldsymbol{\mu}, \boldsymbol{\lambda}, \boldsymbol{v})= & \frac{1}{2} \int_{0}^{T} \sum_{r=1}^{N_{\text {sta }}} W_{r}\left[h(t) *\left(\dot{\mathbf{s}}\left(\mathbf{x}_{\mathbf{r}}, t, \mathbf{m}\right)-\dot{\mathbf{d}}\left(\mathbf{x}_{\mathbf{r}}, t\right)\right)\right]^{2} \mathrm{~d} t \\
& -\int_{0}^{T} \int_{\Omega} \lambda \cdot\left(\rho \partial_{t}^{2} \dot{\mathbf{s}}-\nabla \cdot(\mathbf{C}: \nabla \dot{\mathbf{s}})\right) \mathrm{d}^{3} \mathbf{x} \mathrm{d} t \\
& +\int_{0}^{T} \int_{\Sigma} \boldsymbol{\mu} \cdot\left(\dot{\mathbf{s}}_{+}-\dot{\mathbf{s}}_{-}-\mathbf{m}\right) \mathrm{d}^{2} \mathbf{x} \mathrm{d} t \\
& +\int_{0}^{T} \int_{\Sigma} \boldsymbol{v} \cdot\left(\mathbf{T}_{+}+\mathbf{T}_{-}\right) \mathrm{d}^{2} \mathbf{x} \mathrm{d} t,
\end{aligned}
$$

where $\lambda, \boldsymbol{\mu}$ and $\boldsymbol{v}$ are Lagrange multipliers that depend on space and time, $\dot{\mathbf{s}}_{+}$and $\dot{\mathbf{s}}_{-}$are the velocity fields on each side of the fault and $\mathbf{m}$ is slip velocity on the fault plane $\Sigma$. $\mathbf{T}_{+}$and $\mathbf{T}_{-}$are the tractions on each side of the fault given by $\mathbf{T}_{ \pm}=[\hat{\mathbf{n}} .(C: \nabla \mathbf{s})]_{ \pm}, \hat{\mathbf{n}}_{ \pm}$is the normal vector of the respective side of a fault interface, $W_{r}$ is the weight assigned to the data at the $r$ th station, $h(t)$ is a low-pass filter that downweights the data at frequencies that cannot be modelled accurately with the available crustal velocity model, or that have low signal-to-noise ratio, and $T$ is the total duration of seismograms relative to the earthquake origin time.

We seek $\dot{\mathbf{s}}, \mathbf{m}, \boldsymbol{\lambda}, \boldsymbol{\mu}$ and $\boldsymbol{v}$ such that the Lagrangian $\mathcal{L}$ is minimized. A necessary condition is stationarity of the Lagrangian with respect to its five arguments:

$$
\begin{aligned}
& d \mathcal{L}(\dot{\mathbf{s}}, \mathbf{m}, \boldsymbol{\mu}, \boldsymbol{\lambda}, \boldsymbol{v})=0=\frac{\partial \mathcal{L}}{\partial \dot{\mathbf{s}}} \delta \dot{\mathbf{s}}+\frac{\partial \mathcal{L}}{\partial \mathbf{m}} \delta \mathbf{m}+\frac{\partial \mathcal{L}}{\partial \lambda} \delta \lambda+\frac{\partial \mathcal{L}}{\partial \boldsymbol{\mu}} \delta \boldsymbol{\mu} \\
& +\frac{\partial \mathcal{L}}{\partial \boldsymbol{v}} \delta \boldsymbol{v}, \quad \forall \delta \dot{\mathbf{s}}, \delta \mathbf{m}, \delta \boldsymbol{\lambda}, \delta \boldsymbol{\mu}, \delta \boldsymbol{v} .
\end{aligned}
$$

This implies

$$
\begin{array}{ll}
0=\frac{\partial \mathcal{L}}{\partial \dot{\mathbf{s}}} \delta \dot{\mathbf{s}} & \forall \delta \dot{\mathbf{s}}, \\
0=\frac{\partial \mathcal{L}}{\partial \mathbf{m}} \delta \mathbf{m} & \forall \delta \mathbf{m}, \\
0=\frac{\partial \mathcal{L}}{\partial \lambda} \delta \boldsymbol{\lambda} & \forall \delta \boldsymbol{\lambda}, \\
0=\frac{\partial \mathcal{L}}{\partial \mu} \delta \boldsymbol{\mu} & \forall \delta \boldsymbol{\mu}, \\
0=\frac{\partial \mathcal{L}}{\partial \boldsymbol{v}} \delta \boldsymbol{v} & \forall \delta \boldsymbol{v} .
\end{array}
$$

We now examine these four conditions sequentially. The first stationarity condition (7) is

$$
\begin{aligned}
0=\frac{\partial \mathcal{L}}{\partial \dot{\mathbf{s}}} \delta \dot{\mathbf{s}}= & \int_{0}^{T} \int_{\Omega} \sum_{r} W_{r} \delta\left(\mathbf{x}-\mathbf{x}_{\mathbf{r}}\right)[h(t) *(\dot{\mathbf{s}}(\mathbf{x}, t, \mathbf{m})-\dot{\mathbf{d}}(\mathbf{x}, t))] \\
& \times h(t) * \delta \dot{\mathbf{s}} \mathrm{d}^{3} \mathbf{x} \mathrm{d} t \\
& -\int_{0}^{T} \int_{\Omega} \lambda \cdot\left[\rho \partial_{t}^{2} \delta \dot{\mathbf{s}}-\nabla \cdot(\mathbf{C}: \nabla \delta \dot{\mathbf{s}})\right] \mathrm{d}^{3} \mathbf{x} \mathrm{d} t \\
& +\int_{0}^{T} \int_{\Sigma} \boldsymbol{\mu} \cdot\left(\delta \dot{\mathbf{s}}_{+}-\delta \dot{\mathbf{s}}_{-}\right) \mathrm{d}^{2} \mathbf{x} \mathrm{d} t \\
& +\int_{0}^{T} \int_{\Sigma} \boldsymbol{v} \cdot\left([\hat{\mathbf{n}} \cdot(C: \nabla \delta \dot{\mathbf{s}})]_{+}+[\hat{\mathbf{n}} \cdot(C: \nabla \delta \dot{\mathbf{s}})]_{-}\right) \mathrm{d}^{2} \mathbf{x} \mathrm{d} t
\end{aligned}
$$

Integrating by parts we obtain, after some algebra,

$$
\begin{aligned}
& 0=\frac{\partial \mathcal{L}}{\partial \dot{\mathbf{s}}} \delta \dot{\mathbf{s}}=\int_{0}^{T} \int_{\Omega} \sum_{r} W_{r} \delta\left(\mathbf{x}-\mathbf{x}_{\mathbf{r}}\right)[h *(\dot{\mathbf{s}}-\dot{\mathbf{d}})] \cdot h * \delta \dot{\mathbf{s}} \mathrm{d}^{3} \mathbf{x} \mathrm{d} t \\
& -\int_{0}^{T} \int_{\Omega}\left[\rho \partial_{t}^{2} \lambda-\nabla \cdot(\mathbf{C}: \nabla \lambda)\right] \cdot \delta \dot{\mathbf{s}} \mathrm{d}^{3} \mathbf{x} \mathrm{d} t \\
& -\int_{\Omega}\left[\rho\left(\lambda \cdot \partial_{t}(\delta \dot{\mathbf{s}})-\partial_{t} \lambda \cdot \delta \dot{\mathbf{s}}\right)\right]_{0}^{T} \mathrm{~d}^{3} x \\
& +\int_{0}^{T} \int_{\partial \Omega_{1}} \lambda \cdot[\hat{\mathbf{n}} \cdot(C: \nabla \delta \dot{\mathbf{s}})]-\hat{\mathbf{n}} \cdot(C: \nabla \lambda) \cdot \delta \dot{\mathbf{s}} \mathrm{d}^{2} \mathbf{x} \mathrm{d} t \\
& +\int_{0}^{T} \int_{\partial \Omega_{2}} \lambda \cdot[\hat{\mathbf{n}} \cdot(C: \nabla \delta \dot{\mathbf{s}})]-\hat{\mathbf{n}} \cdot(C: \nabla \lambda) \cdot \delta \dot{\mathbf{s}} \mathrm{d}^{2} \mathbf{x} \mathrm{d} t \\
& +\int_{0}^{T} \int_{\Sigma_{+}} \lambda \cdot[\hat{\mathbf{n}} \cdot(C: \nabla \delta \dot{\mathbf{s}})]-\hat{\mathbf{n}} \cdot(C: \nabla \lambda) \cdot \delta \dot{\mathbf{s}} \mathrm{d}^{2} \mathbf{x} \mathrm{d} t \\
& +\int_{0}^{T} \int_{\Sigma_{-}} \lambda \cdot[\hat{\mathbf{n}} \cdot(C: \nabla \delta \dot{\mathbf{s}})]-\hat{\mathbf{n}} \cdot(C: \nabla \lambda) \cdot \delta \dot{\mathbf{s}} \mathrm{d}^{2} \mathbf{x} \mathrm{d} t \\
& +\int_{0}^{T} \int_{\Sigma} \boldsymbol{\mu} \cdot\left(\delta \dot{\mathbf{s}}_{+}-\delta \dot{\mathbf{s}}_{-}\right) \mathrm{d}^{2} \mathbf{x} \mathrm{d} t \\
& +\int_{0}^{T} \int_{\Sigma} \boldsymbol{v} \cdot\left([\hat{\mathbf{n}} \cdot(C: \nabla \delta \dot{\mathbf{s}})]_{+}+[\hat{\mathbf{n}} \cdot(C: \nabla \delta \dot{\mathbf{s}})]_{-}\right) \mathrm{d}^{2} \mathbf{x} \mathrm{d} t,
\end{aligned}
$$

where $[f]_{0}^{T}=f(T)-f(0)$ for any function $f$. We can simplify this expression by considering the perturbed field which is initially at rest, $\delta \dot{\mathbf{s}}(\mathbf{x}, 0)=\mathbf{0}$ and $\partial_{t} \delta \dot{\mathbf{s}}(\mathbf{x}, 0)=\mathbf{0}$, and satisfies the free surface boundary condition, $\hat{\mathbf{n}} \cdot(C: \nabla \delta \dot{\mathbf{s}})=0$ on $\partial \Omega_{1}$. The absorbing boundary condition can be written as $\hat{\mathbf{n}} \cdot(C: \nabla \delta \dot{\mathbf{s}})=C_{a b s} \delta \ddot{\mathbf{s}}$ on $\partial \Omega_{2}$, the contribution of which we further integrate by parts. The continuity of tractions on the fault surface gives $[\hat{\mathbf{n}} \cdot(C: \nabla \delta \dot{\mathbf{s}})]_{+}=$ $-[\hat{\mathbf{n}} \cdot(C: \nabla \delta \dot{\mathbf{s}})]_{-}$, noting in addition that $\hat{\mathbf{n}}_{+}=-\hat{\mathbf{n}}_{-}$. We then obtain:

$$
\begin{aligned}
0=\frac{\partial \mathcal{L}}{\partial \dot{\mathbf{s}}} \delta \dot{\mathbf{s}}= & \int_{0}^{T} \int_{\Omega} \sum_{r} W_{r} \delta\left(\mathbf{x}-\mathbf{x}_{\mathbf{r}}\right)[h *(\dot{\mathbf{s}}-\dot{\mathbf{d}})] \cdot h * \delta \dot{\mathbf{s}} \mathrm{d}^{3} \mathbf{x} \mathrm{d} t \\
& -\int_{0}^{T} \int_{\Omega}\left[\rho \partial_{t}^{2} \lambda-\nabla \cdot(\mathbf{C}: \nabla \lambda)\right] \cdot \delta \dot{\mathbf{s}} \mathrm{d}^{3} \mathbf{x} \mathrm{d} t \\
& -\int_{\Omega}\left[\rho\left(\lambda \cdot \partial_{t}(\delta \dot{\mathbf{s}})-\partial_{t} \lambda \cdot \delta \dot{\mathbf{s}}\right)\right](T) \mathrm{d}^{3} x \\
& -\int_{0}^{T} \int_{\partial \Omega_{1}} \hat{\mathbf{n}} \cdot(C: \nabla \lambda) \cdot \delta \dot{\mathbf{s}} \mathrm{d}^{2} \mathbf{x} \mathrm{d} t \\
& +\int_{\partial \Omega_{2}}\left[C_{\mathrm{abs}} \lambda \cdot \delta \dot{\mathbf{s}}\right](T) \mathrm{d}^{2} \mathbf{x} \\
& -\int_{0}^{T} \int_{\partial \Omega_{2}}\left(C_{\mathrm{abs}} \dot{\lambda}+\hat{\mathbf{n}} \cdot(C: \nabla \lambda)\right) \cdot \delta \dot{\mathbf{s}} \mathrm{d}^{2} \mathbf{x} \mathrm{d} t \\
& +\int_{0}^{T} \int_{\Sigma}\left[\left(\lambda_{+}-\lambda \lambda_{-}\right) \cdot[\hat{\mathbf{n}} \cdot(C: \nabla \delta \dot{\mathbf{s}})]\right. \\
& -[\hat{\mathbf{n}} \cdot(C: \nabla \lambda) \cdot \delta \dot{\mathbf{s}}]_{+}-[\hat{\mathbf{n}} \cdot(C: \nabla \lambda) \cdot \delta \dot{\mathbf{s}}]_{-} \\
& \left.+\boldsymbol{\mu} \cdot\left(\delta \dot{\mathbf{s}}_{+}-\delta \dot{\mathbf{s}}_{-}\right)\right] \mathrm{d}^{2} \mathbf{x} \mathrm{d} t .
\end{aligned}
$$

We note that the adjoint of convolution is cross-correlation, which we denote by $\star$ :

$$
\int a(t)[h * b](t) \mathrm{d} t=\int[h \star a](t) b(t) \mathrm{d} t
$$

for any pair of functions $a(t)$ and $b(t)$. The first term in eq. (14) can then be written as

$$
\int_{0}^{T} \int_{\Omega} \sum_{r} W_{r} \delta\left(\mathbf{x}-\mathbf{x}_{\mathbf{r}}\right)[h \star h *(\dot{\mathbf{s}}-\dot{\mathbf{d}})] \cdot \delta \dot{\mathbf{s}} \mathrm{d}^{3} \mathbf{x} \mathrm{d} t .
$$

Since the stationarity condition should be valid for all $\delta \dot{\mathbf{s}}$, we get :

$\rho \partial_{t}^{2} \lambda-\nabla \cdot(\mathbf{C}: \nabla \lambda)=\sum_{r} W_{r} \delta\left(\mathbf{x}-\mathbf{x}_{\mathbf{r}}\right) h \star h *(\dot{\mathbf{s}}-\dot{\mathbf{d}})$, 
subjected to the following boundary and terminal conditions:

$$
\begin{aligned}
\hat{\mathbf{n}} \cdot(C: \nabla \lambda) & =0 \quad \text { on } \partial \Omega_{1} \\
\hat{\mathbf{n}} \cdot(C: \nabla \lambda) & =-C_{\text {abs }} \dot{\lambda} \quad \text { on } \partial \Omega_{2} \\
\lambda(\mathbf{x}, T) & =\mathbf{0} \\
\partial_{t} \lambda(\mathbf{x}, T) & =\mathbf{0} \\
\lambda_{+} & =\lambda_{-} \quad \text { on } \Sigma
\end{aligned}
$$

$[\hat{\mathbf{n}} \cdot(C: \nabla \lambda)]_{+}=-[\hat{\mathbf{n}} \cdot(C: \nabla \lambda)]_{-}$on $\Sigma$

and to the following requirement:

$\boldsymbol{\mu}=[\hat{\mathbf{n}} \cdot(C: \nabla \lambda)]_{+}$.

We define the adjoint wavefield as

$\mathbf{s}^{\dagger}(\mathbf{x}, t)=\lambda(\mathbf{x}, T-t)$.

It satisfies the following adjoint wave equation:

$\rho \partial_{t}^{2} \mathbf{s}^{\dagger}-\nabla \cdot\left(\mathbf{C}: \nabla \mathbf{s}^{\dagger}\right)=\mathbf{f}^{\dagger}(\mathbf{x}, t)$

subject to the following adjoint source:

$\mathbf{f}^{\dagger}(\mathbf{x}, t)=\sum_{r=1}^{N_{\text {sta }}} W_{r} \delta\left(\mathbf{x}-\mathbf{x}_{\mathbf{r}}\right)[h \star h *(\dot{\mathbf{s}}-\dot{\mathbf{d}})](T-t)$,

and to the following boundary and initial conditions:

$$
\begin{aligned}
\hat{\mathbf{n}} \cdot\left(C: \nabla \mathbf{s}^{\dagger}\right) & =0 \quad \text { on } \partial \Omega_{1}, \\
\hat{\mathbf{n}} \cdot\left(C: \nabla \mathbf{s}^{\dagger}\right) & =C_{\text {abs }} \dot{\mathbf{s}}^{\dagger} \quad \text { on } \partial \Omega_{2}, \\
\mathbf{s}^{\dagger}(\mathbf{x}, 0) & =\mathbf{0}, \\
\partial_{t} \mathbf{s}^{\dagger}(\mathbf{x}, 0) & =\mathbf{0}, \\
\boldsymbol{s}_{+}^{\dagger} & =\boldsymbol{s}_{-}^{\dagger} \quad \text { on } \Sigma, \\
{\left[\hat{\mathbf{n}} \cdot\left(C: \nabla \boldsymbol{s}^{\dagger}\right)\right]_{+} } & =-\left[\hat{\mathbf{n}} \cdot\left(C: \nabla \boldsymbol{s}^{\dagger}\right)\right]_{-} \quad \text { on } \Sigma .
\end{aligned}
$$

In the adjoint problem, the boundary condition is a locked fault (no slip): the last two equations express the continuity of displacements and tractions of the adjoint field across the fault surface $\Sigma$. We define the adjoint tractions on the '+' side of the fault, $\Sigma_{+}$, by

$\mathbf{T}^{\dagger}(\mathbf{x}, t)=\left(C(\mathbf{x}): \nabla \mathbf{s}^{\dagger}(\mathbf{x}, t)\right) \cdot \hat{\mathbf{n}}(\mathbf{x})$.

Eq. (18) then gives, for any $\mathbf{x} \in \Sigma$,

$\boldsymbol{\mu}=\mathbf{T}^{\dagger}(\mathbf{x}, t)$.

The second stationarity condition $(8)$ is

$0=\frac{\partial \mathcal{L}}{\partial \mathbf{m}} \delta \mathbf{m}=\frac{\partial \chi}{\partial \mathbf{m}} \delta \mathbf{m}-\int_{0}^{T} \int_{\Sigma} \boldsymbol{\mu} \cdot \delta \mathbf{m} \mathrm{d}^{2} \mathbf{x} \mathrm{d} t$.

Considering eq. (24), we obtain a useful expression for the gradient of the cost function:

$\frac{\partial \chi}{\partial \mathbf{m}} \delta \mathbf{m}=\int_{0}^{T} \int_{\Sigma} \mathbf{T}^{\dagger}(\mathbf{x}, T-t) \cdot \delta \mathbf{m} \mathrm{d}^{2} \mathbf{x} \mathrm{d} t$.

The third stationarity condition (9) is

$0=\frac{\partial \mathcal{L}}{\partial \lambda} \delta \boldsymbol{\lambda}=\int_{0}^{T} \int_{\Omega} \delta \boldsymbol{\lambda} \cdot\left[\rho \partial_{t}^{2} \dot{\mathbf{s}}-\nabla \cdot(\mathbf{C}: \nabla \dot{\mathbf{s}})\right] \mathrm{d}^{3} \mathbf{x} \mathrm{d} t$.

Since the above equation should be valid for all $\delta \lambda$, we obtain

$\rho \partial_{t}^{2} \dot{\mathbf{s}}-\nabla \cdot(\mathbf{C}: \nabla \dot{\mathbf{s}})=\mathbf{0}$,

which is the forward wave equation.

Similarly, the fourth (10) and fifth (11) stationarity conditions yield the slip and traction boundary conditions on the fault for the forward problem; for any $\mathbf{x} \in \Sigma$,

$\mathbf{m}(\mathbf{x}, t)=\dot{\mathbf{s}}_{+}(\mathbf{x}, t)-\dot{\mathbf{s}}_{-}(\mathbf{x}, t)$,

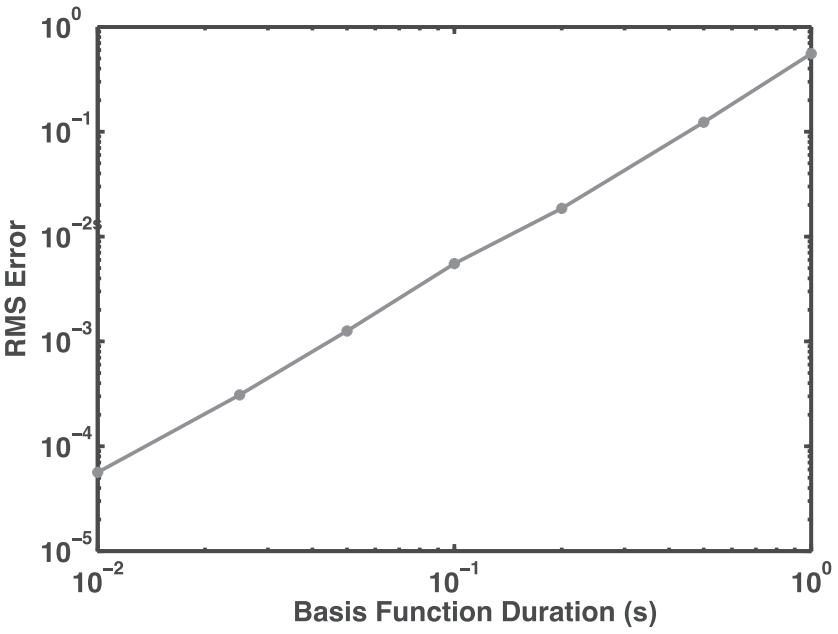

Figure 2. Root-mean-squared (RMS) difference between the data and synthetics as a function of the time step $\Delta t_{m}$ of the temporal basis functions that discretize the slip velocity. The data are computed based on a very finely discretized source to represent a continuum source. A time step of $0.1 \mathrm{~s}$ explains $99 \%$ of the data. In all these simulations, the data are filtered using a low-pass fourth-order Butterworth filter with the corner frequency at $1 \mathrm{~Hz}$.

$\mathbf{T}_{+}=-\mathbf{T}_{-}$.

In common practice, seismograms are band-pass filtered prior to source inversion. In previous adjoint point source inversion implementations (Kremers et al. 2011; Hjörleifsdóttir et al. 2007; Kim et al. 2011) based on pre-filtered data, the model actually represents a filtered version of the complete source model. The approach described here handles the temporal filter in a novel way: the forcing term (eq. 21) for the adjoint wave equation involves a cross-correlation of the filter and the filtered residuals. This is the result of the Lagrangian based on the filtered waveform residuals (eq. 5). In eq. (21), the cross-correlation operation is introduced in the data space. Alternatively, one can introduce the cross-correlation in the model space, in which case the integrand of eq. (26) will be a cross-correlation of the filter and adjoint tractions. Whether the cross-correlation is introduced in the data space or the model space, our formulation allows to retrieve the filtered version of the source.

\subsection{Discrete formulation and model parametrization}

The simplest possible parametrization of the model is adopted here. The fault surface is decomposed into a mesh of $N_{\text {fault }}$ nonoverlapping fault elements of size $\Delta x_{m}$. The time axis is decomposed into $N_{t}$ regular time intervals of size $\Delta t_{m}$. The slip rate is represented by a piecewise constant distribution in space and time, that is, slip rate is constant inside each fault element and each time interval. This implies that the unknowns in our inverse problem are essentially the average slip rates within spatio-temporal cells of dimensions $\Delta x_{m}^{2} \times \Delta t_{m}$.

We set the temporal resolution of the model parametrization, $\Delta t_{m}$, such that, in a representative earthquake scenario simulation, an RMS misfit lower than $1 \%$ is achieved between low-pass filtered ground motions computed from a continuum source and from its temporally discrete version. In practice, the continuum source is also discrete, but described with a time step much smaller than $\Delta t_{m}$. For a specific source scenario described in a later section, we find that the RMS criterion is achieved with $\Delta t_{m}=0.1 / f_{c}$, where $f_{c}$ is the upper cut-off frequency of the data filter (Fig. 2). 
We set the spatial resolution of the model parametrization, $\Delta x_{m}$, to be much smaller than the minimum wavelength at the cut-off frequency, $\lambda=c_{S} / f_{c}$ (Liu \& Archuleta 2004), where $c_{S}$ is the shear wave speed. We carry out the wave propagation simulations using the spectral element method (SEM) code SPECFEM3D (Komatitsch et al. 2010). Adequate accuracy is obtained by setting a spectral element size comparable to the minimum wavelength $\lambda$ and a polynomial order of four, that is, the number of Gauss-LobattoLegendre nodes per spectral element edge is $N_{\mathrm{GLL}}=5$. The slip velocity model is represented at all the spectral nodes, so the average spatial resolution of the model is $\Delta x_{m} \approx \lambda /\left(N_{\mathrm{GLL}}-1\right)$.

\subsection{Verification of the adjoint field computation}

Any 3-D wave propagation code may be used in our source inversion method, if the adjoint simulation capabilities are implemented. In particular, it is important to verify that the wave propagation code passes the dot-product test (Claerbout \& Fomel 2008) to guarantee that the computation of the adjoint field is implemented correctly. The test amounts to verifying that eq. (4) holds for arbitrary $\mathbf{m}^{\prime}$ and $\mathbf{d}^{\prime}$. The test involves two inner products, one in the data space and one in the model space. The inner product in the data space is the one emerging from the definition of the cost function (eq. 2). The inner product in the model space is

$\left\langle\mathbf{G}^{\dagger} \mathbf{d}^{\prime}, \mathbf{m}^{\prime}\right\rangle=\Delta t_{m} \sum_{p=0}^{N_{t}} \int_{\Sigma} m_{p}^{\prime}(\mathbf{x}) \tau_{p}^{\prime}(\mathbf{x}) \mathrm{d}^{2} \mathbf{x}=\tau^{\prime \dagger} W_{m} m^{\prime}$,

where $\tau_{p}^{\prime}$ is the discrete version of the adjoint field, $\mathbf{G}^{\dagger} \mathbf{d}^{\prime}$, at the $p$ th time step and $W_{m}$ is the weighting matrix introduced by the spectral element quadrature. We verified the implementation of our adjoint in the spectral element code by computing the inner product in the model space and finding it to be the same as that in the data space.

\section{COMPUTATIONAL COST OF ADJOINT METHOD COMPARED TO PRE-COMPUTED GREEN'S FUNCTIONS APPROACH}

Here we compare the computational cost of our adjoint source inversion method to that of the prevailing GF approach. The computational cost is defined here as the computational complexity quantified by the total number of floating-point operations. The actual performance on modern-day computers depends also on other factors including cache or CPU pipeline optimization, I/O density and interprocessor communication. However, closed-form relations can be conveniently derived with a metric based on arithmetic operations which, as we have found in practice, allow an adequate comparison between two methods implemented on the same computer across a wide range of application scenarios.

In Appendix A, we develop formulas relating the cost ratio of the two methods, $\Gamma_{\mathrm{GF}} / \Gamma_{\text {adj }}$, to algorithmic parameters such as the number of fault cells $N_{\text {fault }}$, the number of CG iterations $N_{\text {iter, }}$, the number of stations $N_{\text {sta }}$, etc. The cost ratio is made of two contributions, the ratio of number of operations per CG iteration and the relative overhead of pre-computing Green's functions in the GF approach:

$\frac{\Gamma_{\mathrm{GF}}}{\Gamma_{\mathrm{adj}}}=\frac{C_{\mathrm{conv}}}{C_{\mathrm{SEM}}}+\frac{\Gamma_{\mathrm{GF}}^{\mathrm{oh}}}{\Gamma_{\mathrm{adj}}}$,

where $\Gamma_{\mathrm{GF}}$ is the total computational cost of an inversion with the GF approach, $\Gamma_{\text {adj }}$ is the total computational cost of an inversion with the adjoint approach, $C_{\text {conv }}$ is the computational cost for convolutions between the Green's functions and the slip rate time-series, $C_{\mathrm{SEM}}$ is the computational cost of a single 3-D SEM wave propagation simulation and $\Gamma_{\mathrm{GF}}^{\mathrm{oh}}$ is the overhead cost of pre-computing the Green's functions. We develop the following order-of-magnitude estimates of these two contributions as a function of three non-dimensional parameters that encapsulate information about the fault geometry, network geometry and maximum frequency:

$\frac{C_{\mathrm{conv}}}{C_{\mathrm{SEM}}}=O(1) \frac{\lambda W}{\Delta x_{s}^{2}}$

and

$\frac{\Gamma_{\mathrm{GF}}^{\mathrm{oh}}}{\Gamma_{\mathrm{adj}}}=O\left(10^{-1}\right) \min \left(\frac{\mathrm{WL}}{\lambda^{2}},\left(\frac{L}{\Delta x_{s}}\right)^{2}\right)$.

where $\lambda$ is the shortest wavelength, inversely proportional to the high frequency cut-off $f_{c}$ considered in the inversion; $\Delta x_{s}$ is the average spacing between stations; and $L$ and $W$ are the rupture length and width, respectively.

Eqs (32)-(34) provide a useful guideline to determine which approach is the most efficient for a given earthquake size, network density, and target frequency. In particular, if the wave field is recorded without aliasing on a dense seismic network with $\Delta x_{s} \approx$ $\lambda$, then

$\frac{C_{\text {conv }}}{C_{\mathrm{SEM}}}=O(1) \frac{W}{\lambda}$

and

$\frac{\Gamma_{\mathrm{GF}}^{\mathrm{oh}}}{\Gamma_{\mathrm{adj}}}=O\left(10^{-1}\right) \frac{W L}{\lambda^{2}}$.

We first compare the cost per iteration of both approaches. In the source inversion of large, $M_{\mathrm{w}} \geq 7$ earthquakes, the cut-off frequency is typically lower than $1 \mathrm{~Hz}$, and the corresponding wavelength $\lambda$ is comparable to or somewhat smaller than the average spacing of current seismic networks (which is typically larger than $10 \mathrm{~km}$ ): $\lambda / \Delta x_{\mathrm{s}} \lesssim 1$. Moreover, $W / \Delta x_{\mathrm{s}} \lesssim 1$. Considering these numbers and eq. (33), we get $C_{\text {conv }} / C_{\mathrm{SEM}}<1$ : in the currently typical source inversion scenarios, the GF approach has a significantly smaller cost per iteration than the adjoint approach based on SEM. However, as progress continues to be made in earthquake seismology to exploit higher frequencies of the wave field $(W / \lambda \gg 1)$, recorded without aliasing on dense seismic networks, eq. (35) shows that the full advantage of the adjoint approach, in terms of a lower cost per iteration, will eventually be realized.

Even in situations in which the cost per iteration is lower in the GF approach, the adjoint approach can have a relative advantage due to the overhead of pre-computing Green's functions. Typically, rupture aspect ratios are in the range $W / L=0.1-1$ and the cut-off wavelength is a small to moderate fraction of the rupture length, $L / \lambda=O(10)$; hence $L W / \lambda^{2}=O(10-100)$. For station spacings typical of existing seismic networks, $L / \Delta x_{s}=O(1-10)$. Considering these numbers, eq. (34) gives $\Gamma_{\mathrm{GF}}^{\mathrm{oh}} / \Gamma_{\mathrm{adj}}=O(0.1-10)$, which shows that, even in coarse networks, the adjoint approach can be more efficient than the GF approach in some situations. Such situations include ruptures that are very long compared to the average station spacing, for instance $M_{\mathrm{w}}>7$ earthquakes recorded on networks with $20 \mathrm{~km}$ average spacing. For a very dense network with $\Delta x_{s} \approx$ $\lambda$, for frequencies up to $1 \mathrm{~Hz}$ or higher $(\lambda<3.6 \mathrm{~km})$ and $M_{\mathrm{w}}>6.2$, typically $L W / \lambda^{2}>10$ and eq. (36) shows that the adjoint approach is more advantageous, especially for large ruptures and high frequencies. 


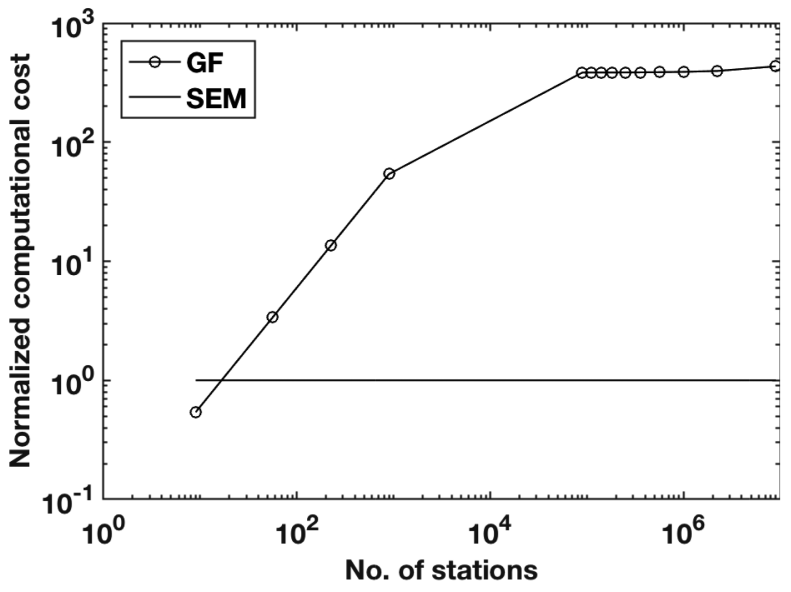

Figure 3. Comparison of the computational cost of the adjoint method based on the spectral element method (SEM) and the method with precomputed Green's functions for a typical $M_{\mathrm{W}} 7.0$ earthquake scenario with the $40 \mathrm{~km} \times 15 \mathrm{~km}$ rupture area, a 3-D velocity model, data low-pass filtered below $1 \mathrm{~Hz}$, a fixed domain size extending up to approximately 2 fault lengths away from the source, and regular station spacing. The cost is normalized by the computational cost of SEM. The cost of the adjoint method does not depend on the number of stations, while the cost of the GF approach increases drastically until it is no longer advantageous to exploit reciprocity to compute the Green's functions. Note that several inversions may be performed with the same pre-computed Green's functions, in which case the cost per inversion will be decreased accordingly. Still, SEM approach will become beneficial for a large enough number of stations.

As a concrete example of how eqs (A7) and (A8) in Appendix A can be used to decide which approach to use, Fig. 3 shows the cost ratio as a function of the number of stations for a specific earthquake scenario considered in a later section. The rupture dimensions are $40 \mathrm{~km}$ along-strike and $15 \mathrm{~km}$ along-dip, typical of a $M_{\mathrm{w}} 7.0$ strikeslip earthquake. Ground motions up to approximately two fault lengths away from the source are included, low-passed filtered below $1 \mathrm{~Hz}$. In this example, the GF approach is more expensive than the adjoint approach if more than a few tens of stations are considered. Also, in all examples with heterogeneous media shown in Section 4, the adjoint approach is more advantageous than the GF approach. When the overhead of the GF approach dominates its cost, it is advantageous to use the adjoint approach if

$\min \left(N_{\text {fault }}, \frac{3}{2} N_{\text {sta }}\right)>N_{\text {iter }}=O(10 \sim 100)$.

\section{IMPACT OF CRUSTAL STRUCTURE UNCERTAINTIES ON THE SOURCE INVERSION}

\subsection{Reference inversions with known 3-D velocity model}

We show here that our source inversion procedure works, in the sense that it reconstructs well the source in the ideal case in which the 3-D velocity model is perfectly known. We do not add any noise to the data. We refer to these inversions as 'reference inversions'.

Let us consider as an earthquake scenario a Haskell source, which is a steady slip pulse with a square slip-rate time function on a vertical strike-slip fault. The rise time is $1 \mathrm{~s}$ and the rupture speed is $2.9 \mathrm{~km} \mathrm{~s}^{-1}$. The fault dimensions are $40 \mathrm{~km} \times 15 \mathrm{~km}$. The stations extend about two fault lengths away on each side of the fault, giving a coverage of $200 \mathrm{~km} \times 160 \mathrm{~km}$. A fourth-order low-pass Butterworth
Table 1. Parameters of the three random velocity models (A, B and C) with the von Karman correlation function.

\begin{tabular}{cccc}
\hline & $\begin{array}{c}\text { Correlation Length }\left(R_{\mathrm{c}}\right) \text { in } \\
\mathrm{km}\end{array}$ & $\begin{array}{c}\text { Standard deviation } \\
(\sigma)\end{array}$ & $\begin{array}{c}\text { Hurst exponent } \\
(v)\end{array}$ \\
\hline Model A & 5 & $5 \%$ & 0.0 \\
Model B & 0.5 & $5 \%$ & 0.0 \\
Model C & 5 & $1 \%$ & 0.0 \\
\hline
\end{tabular}

filter with a corner frequency of $1 \mathrm{~Hz}$ is applied to the data. The fault is embedded in a 3-D velocity model with lateral heterogeneities superimposed on a homogeneous half-space. The average P-wave velocity is $5.6 \mathrm{~km} \mathrm{~s}^{-1}$, S-wave velocity is $3.2 \mathrm{~km} \mathrm{~s}^{-1}$ and density is $2670 \mathrm{~kg} \mathrm{~m}^{3}$. The velocity heterogeneities have the Gaussian probability distribution and von Karman spatial correlation function with correlation length $R_{c}$, standard deviation $\sigma$, and Hurst exponent $H$ given as 'model A' in Table 1 (Fig. 4). We assume $V_{s}=V_{p} / \sqrt{3}$. The parameters for model A are representative of the velocity heterogeneity found in the San Francisco Bay Area by Hartzell et al. (2010).

We consider two different station densities: a 'dense network' with station spacing of $1 \mathrm{~km}$ and a 'coarse network' with station spacing of $20 \mathrm{~km}$ (Fig. 5). For the fault dimensions and discretization considered here, slip inversion using the coarse network is an underdetermined problem, whereas the dense network yields an overdetermined problem. The dense network setup is motivated by emergent concepts for space-based earthquake observation systems (Michel et al. 2013) and the block-by-block measurements using low cost MEMS sensors (Clayton et al. 2012) that could provide ground-motion recordings at every few hundreds of meters. The coarse network is representative of the average station spacing of the current Japanese seismic networks (Okada 2013). Performing an inversion for the dense network would be quite challenging for the currently available source inversion codes.

Fig. 6 shows the results of the inversions based on the coarse and dense networks, after 50 iterations (fixed based on Somala et al. 2014). We only show here the strike-component of slip rate as the input model does not have any dip-component of slip rate. As our model contains both components of slip rate without any constraint on the rake angle, we do observe (not shown here) some leakage of slip into the dip-component but its amplitude was an order of magnitude smaller than the actual slip in this case. The quality of the recovered source is comparable to that found with a similar inversion approach but in homogeneous media by Somala et al. (2014). In particular, the recovery of the first-order source properties, such as rise time, peak slip velocity and rupture speed, is adequate. The spatial distribution of slip rate is sharper in the source model inferred from the dense data set. This illustrates the intuitive idea that source recovery improves with the increased number of sensors. Such an inversion including 3-D heterogeneity of the velocity model from recordings of a dense network ( $\sim 32000$ stations) may not be practically feasible with the pre-computed Green's function approach.

\subsection{Effect of uncertainties in the 3-D velocity model on source recovery}

We discuss here the effects of ignoring the heterogeneity of the crustal velocity model by comparing the reference inversions to inversions that adopt the background homogeneous velocity model. In this comparison we use the same number of $\mathrm{CG}$ iterations as in the reference inversions. The goal is to determine whether or not 
(a)

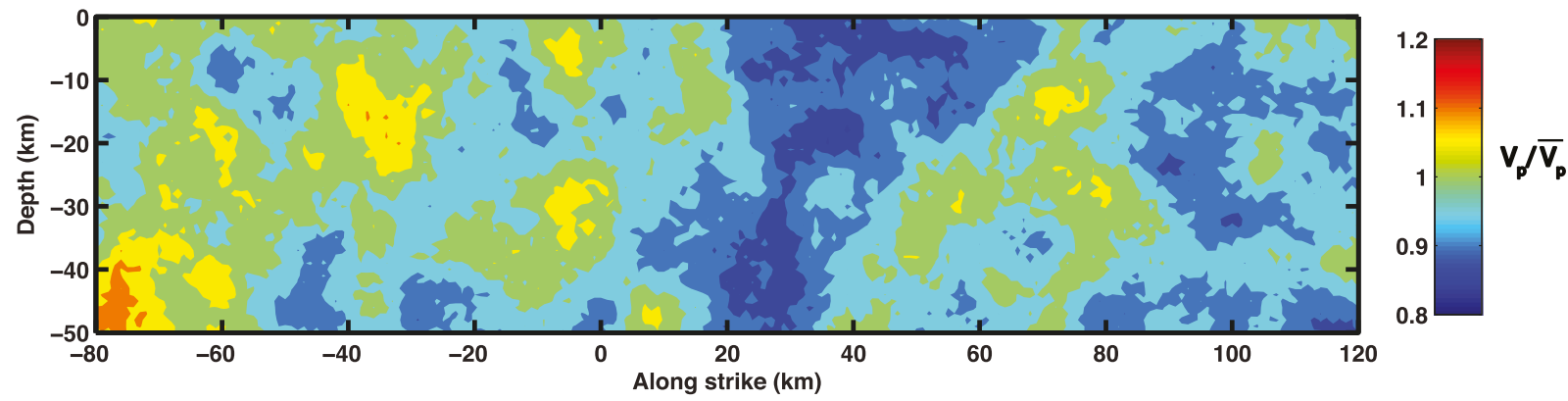

(b)

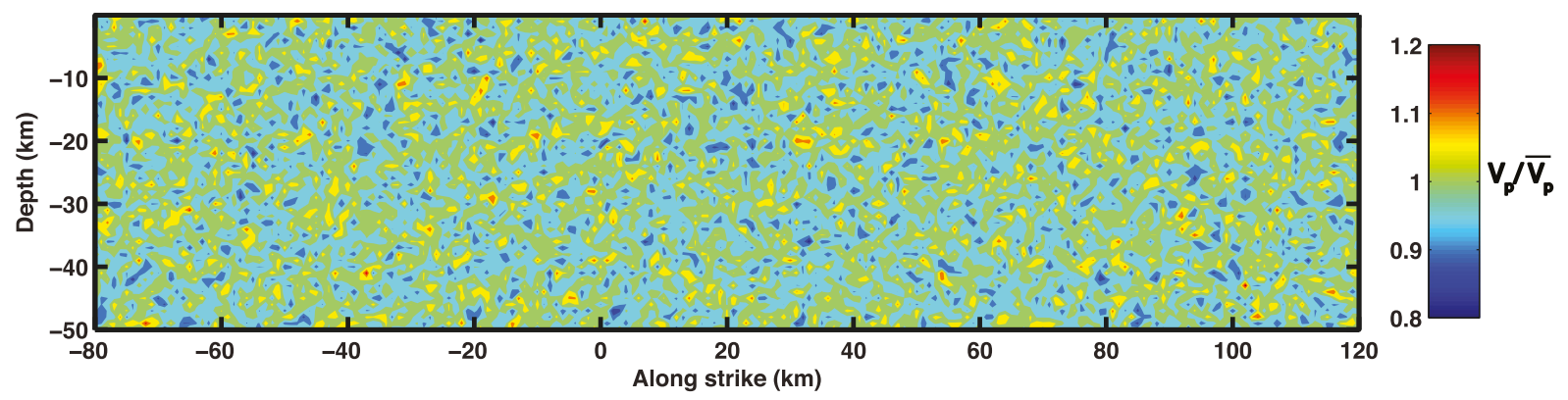

(c)
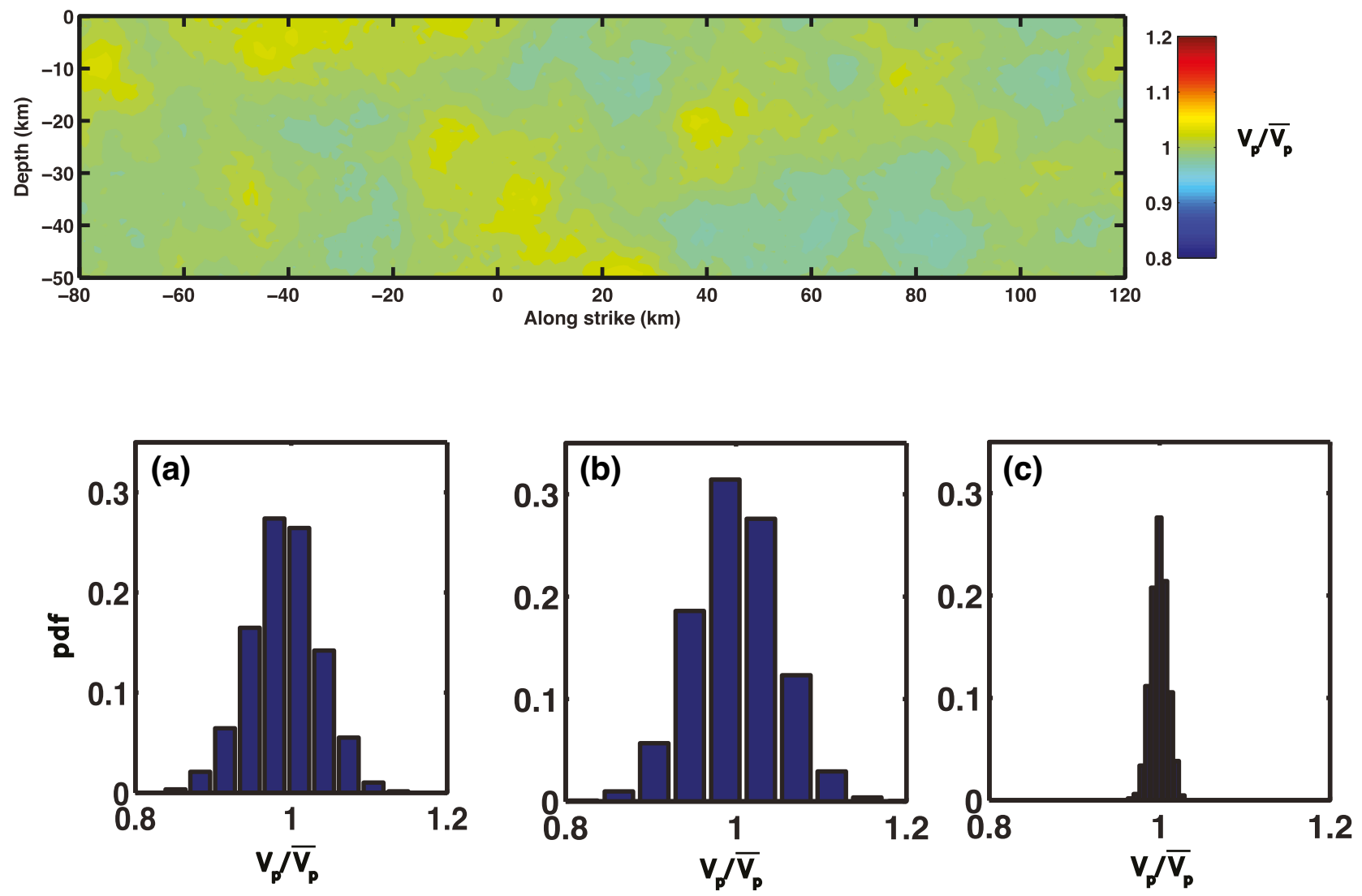

Figure 4. Spatial distribution of the $P$-wave velocity $\left(V_{\mathrm{p}}\right)$, normalized by its mean $\left(5.6 \mathrm{~km} \mathrm{~s}^{-1}\right)$, on a cross-section parallel to the fault plane, for three velocity models A, B and C (from top to bottom) with random heterogeneity described in Table 1. Histograms for each model are shown at the bottom.

one can invert for the slip rate adequately given the typical level of uncertainty in currently available velocity models.

Assuming the heterogeneities in model A are unknown, inversions are carried out in a homogeneous half-space corresponding to the mean velocity of model A. The results for the coarse and dense networks are shown in Fig. 7(a). The main aspects of the source are adequately recovered, such as the overall rupture speed. However, compared to the results of the reference inversions (Fig. 6), there is more significant distortion of the rupture front and smearing of the pulse leading to overestimation of the rise time, especially in the 
(a)

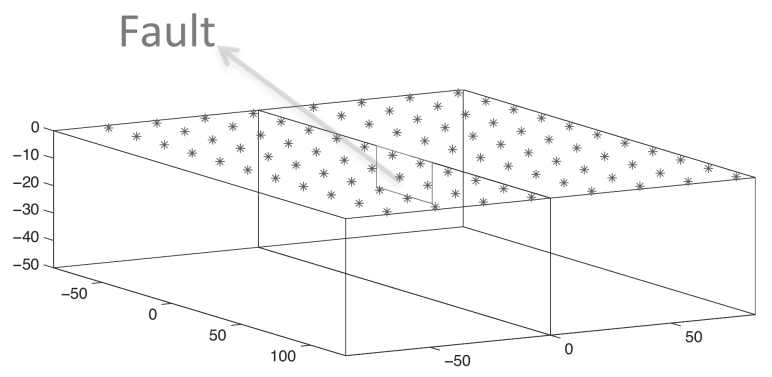

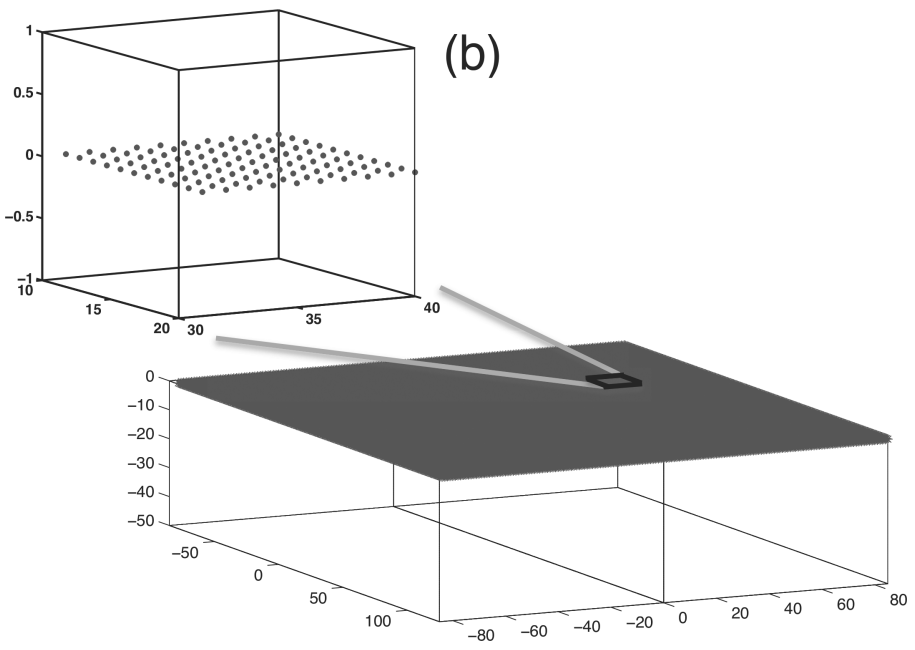

Figure 5. Fault and station geometry for an $M_{\mathrm{w}} 7$ earthquake scenario and two hypothetical networks with (a) $20 \mathrm{~km}$ and (b) $1 \mathrm{~km}$ station spacing. The numbers of stations for the networks are 88 and 32160 , respectively. The fault $(40 \mathrm{~km} \times 15 \mathrm{~km})$ is in the XZ plane. The domain is symmetric with respect to the XZ-plane. Stars indicate station locations. The inset shows a zoomed portion of a $10 \mathrm{~km} \times 10 \mathrm{~km}$ area. The number of stations in the inset is larger than the total number of stations in the $20 \mathrm{~km}$ network.
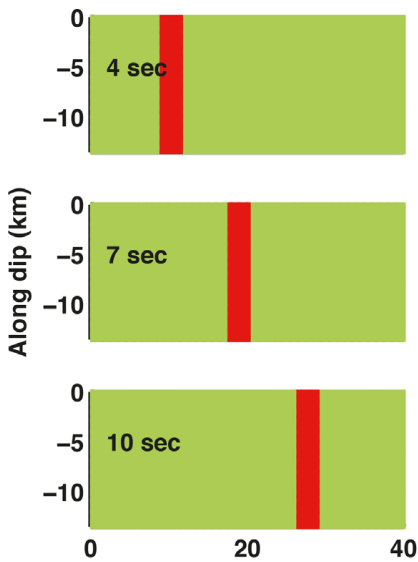
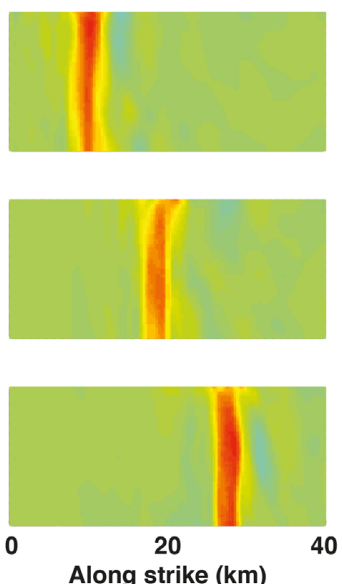
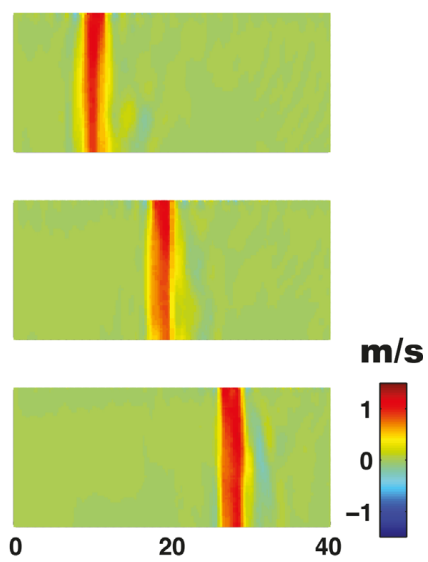

Figure 6. Results from the reference inversions. The data are generated in the 3-D heterogeneous velocity model A and source inversion is performed assuming the same heterogeneous velocity model. The left column shows snapshots of the input slip rate at three different times. The middle and right columns show the inverted slip rate from the data of the $20 \mathrm{~km}$ network and the $1 \mathrm{~km}$ network, respectively. Note that the dense network allows for a better source recovery. It would be challenging for current source inversion codes and methodologies to do this inversion for the dense network with 32160 stations.

coarse network inversion. The denser network still resolves the rise time better than the coarse network, but it has a stronger tendency to bias the distribution of slip rate towards the shallowest portions of the fault at early times. This indicates that a denser network may not improve all aspects of a source inversion if the uncertainties of the velocity structure have the statistical properties assumed here (standard deviation of $5 \%$ and von Karman correlation function with Hurst exponent near zero and correlation length of $5 \mathrm{~km}$ ).

We repeat this exercise for two other 3-D heterogeneous velocity models: one with a much shorter correlation length (model B) and one with a smaller standard deviation (model $\mathrm{C}$ ). The average $P$ wave velocity and $S$-wave velocity are the same for all models. The main parameters of the three models are summarized in Table 1. Typical cross-sections and histograms of the velocity models are shown in Fig. 4. For the earthquake scenarios generated in models $\mathrm{B}$ and $\mathrm{C}$, the inversions assuming the same heterogeneous velocity model as the forward scenario yield slip rates qualitatively similar (not shown) to those obtained in the reference inversions (Fig. 6). In both cases, the dense network enables a better recovery of slip rate than the coarse network. However, the results of the inversions carried out using the background homogeneous velocity model (Figs $7 \mathrm{~b}$ and c, respectively) have visible imperfections compared to the reference inversion (Fig. 6). In the inversion based on the coarse network data, comparing the second columns of Figs 7(a)-(c) shows that ignoring heterogeneities widens the slip rate pulse when both the standard deviation and correlation length of the heterogeneity are relatively high. A factor of 5 reduction in either of those parameters characterizing heterogeneity allows for better resolution of the pulse width, even at depth, but the amplitude of the slip rate is underestimated. In the inversion based on the dense network data, when the heterogeneity of the velocity model is unknown, curving or bending of the recovered pulse is observed below a certain depth. Such curving seems to swing from one side to the other (Figs 7a and c) depending on the amount of the standard deviation for a particular correlation length of the unknown heterogeneity of the medium. The comparison between target seismograms and synthetic waveforms from coarse and dense networks, for all inversions considered in this study, is presented in Appendix B. 


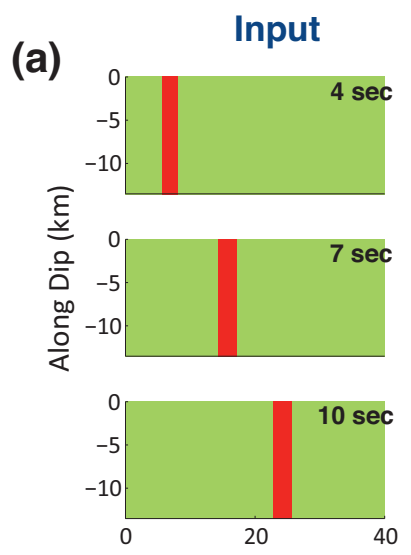

20 km-network
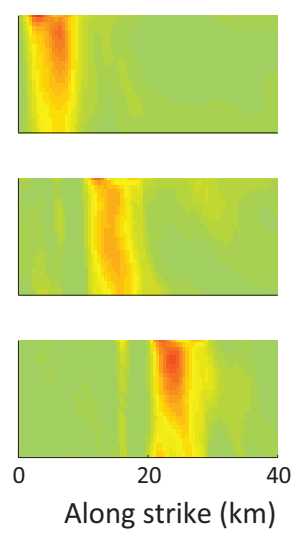

Along strike $(\mathrm{km})$

(b)

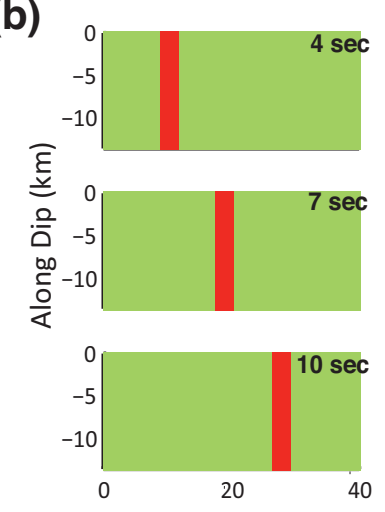

(c)
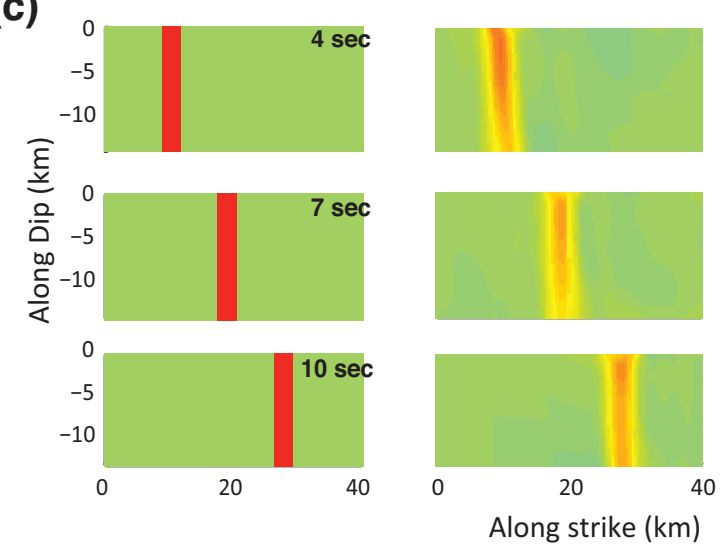
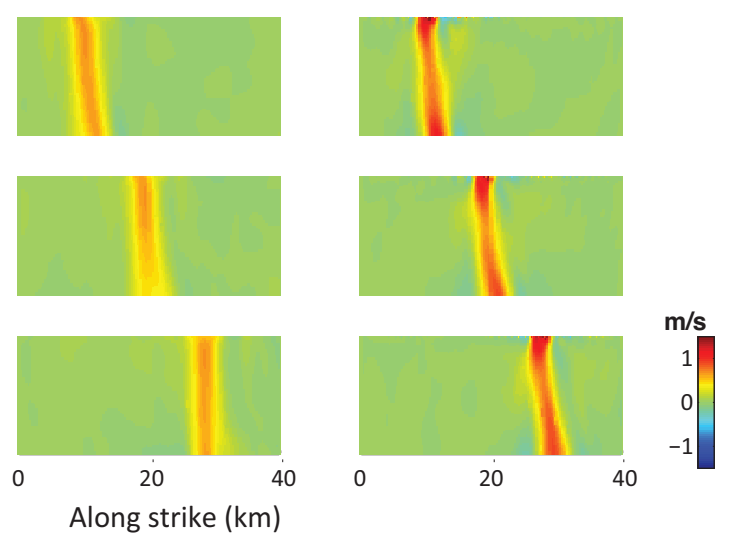

Along strike $(\mathrm{km})$
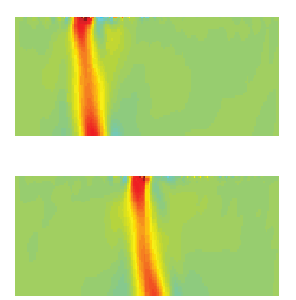

\section{1 km-network}
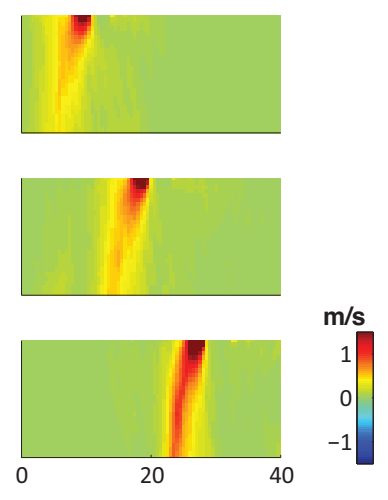
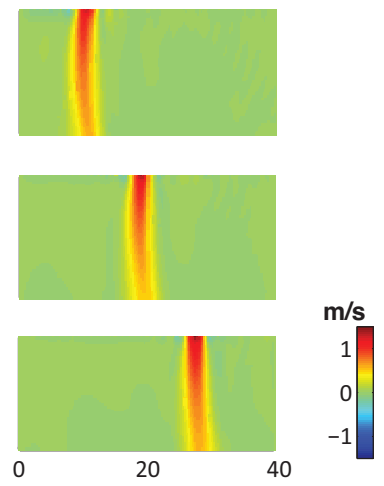

Figure 7. (a) Inversion results similar to Fig. 6, with the data generated in velocity model A but the source inversion based on the background homogeneous velocity model. The inverted slip pulses are distorted (bending in the dense network and widening of the pulse in the sparse network) compared to the reference inversions shown in Fig. 6. (b) Inversion results but with data generated in velocity model B (the source inversion still assumes the homogeneous background velocity model). The pulses recovered with the dense network are distorted while those of the sparse network are qualitatively similar to results of an inversion based on the true velocity model (not shown). The slip rates are better recovered for the dense network. (c) Inversion results with data generated in model C. For both networks, the inverted pulses are qualitatively similar to the results of inversions based on the true velocity model (not shown). Again, the slip rates are better recovered for the dense network.

Fig. 8(a) shows the normalized RMS error between the inverted model and the true model as a function of iteration number for the inversion where the synthetic data are generated using the heterogeneous velocity model $\mathrm{A}$ and the inversion is performed using the background velocity model. As the iterations proceed, the RMS error in model space first decreases and then increases. This increase is faster for the dense network than for the coarse network. A comparison of the sliprate at a particular instance of time after different numbers of iterations (Fig. 8b) shows that despite the increasing RMS model error, the recovery of sliprate continues to improve in the deeper portions of the fault. However, sliprate is increasingly mapped to adjacent fault regions (described earlier as 'curving or 
(a)
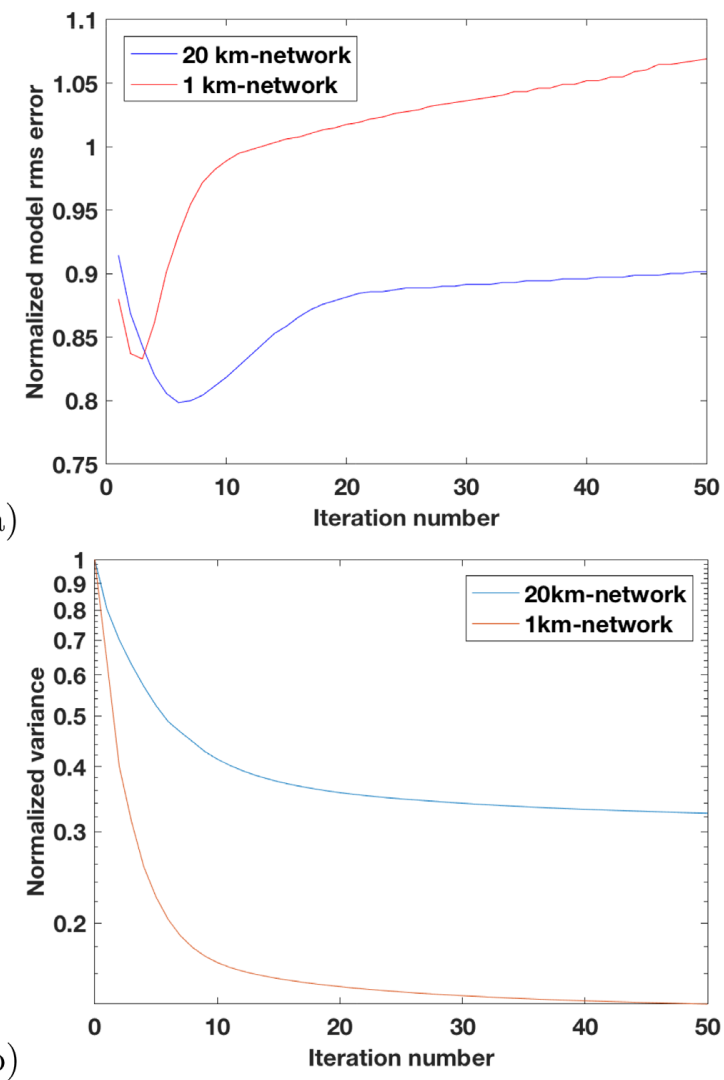

20 km-network
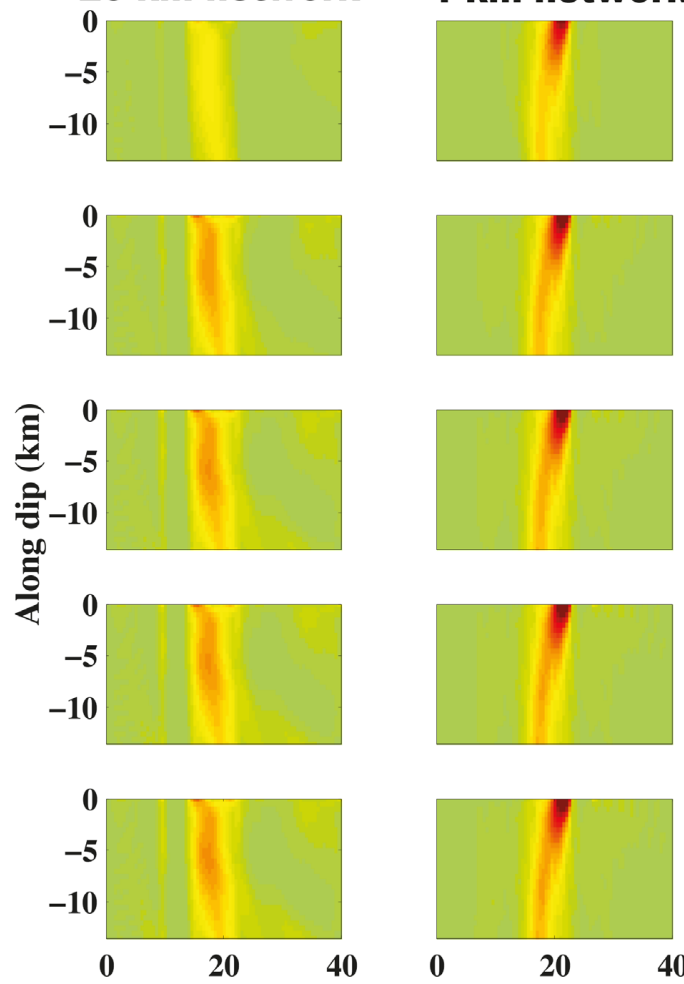

Along strike (km)

(c)

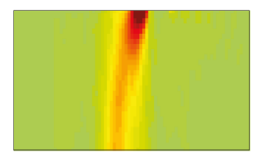

1 km-network
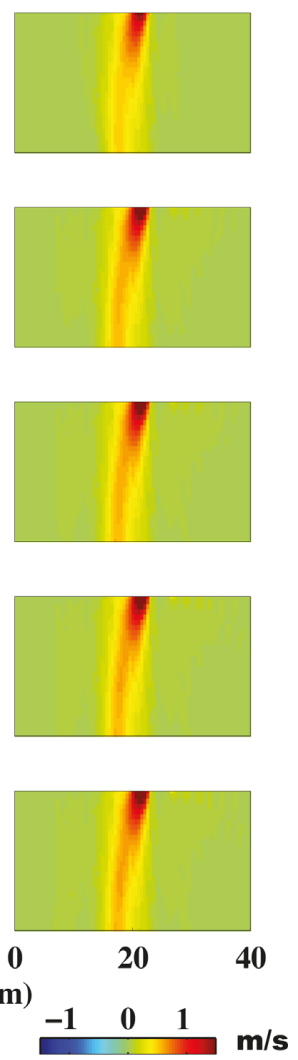

Figure 8. (a) Normalized RMS error in model space, (b) normalized variance in data space and (c) evolution of inverted model with CG iterations, for the worst case in this study. As the iterations progress, the RMS error in model space first decreases and then increases. This increase is faster for the dense network than for the coarse network. Despite the increasing RMS model error, the recovery of slip rate continues to improve in the deeper portions of the fault. Nevertheless, the normalized variance in data space continuously decreases for both networks.

bending of the recovered pulse'), which increases the overall model error. Irrespective of the behaviour of the model space error, the data space error (the variance of the data residuals) decreases monotonically as a function iteration number for both the dense and coarse networks (Fig. 8c).

An explanation for the differences in the effect of velocity model uncertainties on source inversion across the different velocity models considered here could be obtained by examining their power spectra (Fig. 9). The normalized power spectral density of a von Karman distribution is given by

$P(k) \sim \frac{\sigma^{2}\left(4 \pi R_{c}^{2}\right)^{3 / 2}}{\left(1+\left(k R_{c}\right)^{2}\right)^{3 / 2}}$,

where $k$ is the wavenumber. We map the wavenumber into frequency (Frankel \& Clayton 1986) using $2 \pi f=k V_{s}$, where $V_{s}$ is the shear wave speed. The heterogeneities in models $\mathrm{B}$ and $\mathrm{C}$ have low spectral amplitude in the frequency band of inversion $(f<1 \mathrm{~Hz})$, at least one order of magnitude lower than that of model A, and hence they have less impact on the quality of the source inversion.

Our formulation of the inverse problem assumes implicitly that the noise is Gaussian and uncorrelated. In a source inversion based on an incorrect velocity model, the major source of noise is the wavefield scattered by the unknown heterogeneities. This noise can be correlated over length scales comparable to the correlation length of the heterogeneities. One might hypothesize that the spatial correlation of the scattered wavefield affects source inversions that assume uncorrelated noise if its correlation length is larger than the station spacing, and our results support this intuition. In model B, in which the effective correlation length is $2 \pi R_{c} \sim 3.14 \mathrm{~km}$, the coarse network ( $20 \mathrm{~km}$ spacing) may not see this noise correlation, but the dense network (1 km spacing) might (Fig. 7b). For model A, however, both networks sense this correlation, because their station spacing is shorter than the effective correlation length of the medium, $2 \pi R_{c} \sim 31.4 \mathrm{~km}$ (Fig. 7a). Yagi \& Fukahata (2008), Hallo \& Gallovič (2016) and Duputel et al. (2015) have discussed how to better handle the noise correlation induced by uncertainties in the velocity model.

The heterogeneities have the same correlation length in model $\mathrm{C}$ as in model A, but lower amplitudes. The slip pulses inferred in model C (Fig. 7c) have closer resemblance to the reference inversion (Fig. 6) than those inferred in model A (Fig. 7a). This is consistent with the expectation that the effects of the noise correlation can be mitigated by reducing the amplitude of the uncertainties in the velocity model, even if the noise correlation is not accounted for in the inversion technique. Similar work for the layered velocity model was done by Gallovič et al. (2015) in the context of L'Aquila earthquake, where the authors examined the influence of smoothing parameter and emphasized the usage of exact Greens functions with weak smoothing. When the velocity structure is poorly known and dense data sets are available, one may consider a joint inversion of the source and structure (Akcelik et al. 2003). 


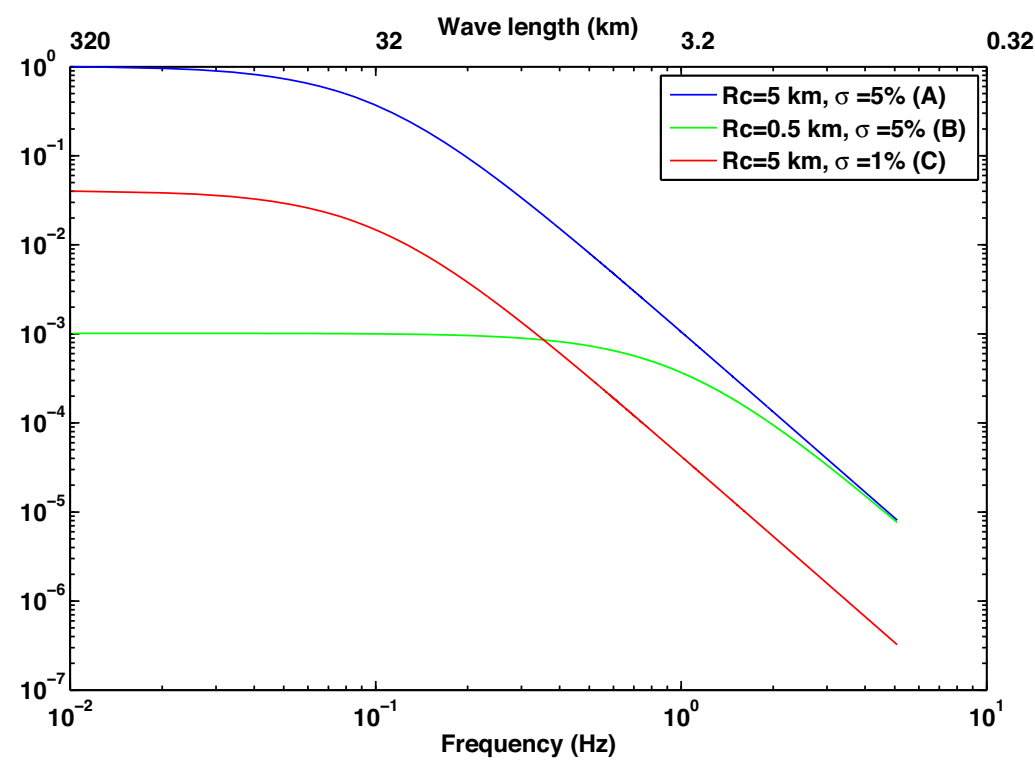

Figure 9. Power spectrum of the three heterogeneous velocity models considered, normalized by the peak value of model A.

\subsection{Validity of our study for other rise times}

Although we consider only one scenario with rise time of $1 \mathrm{~s}$, we expect our findings to be valid for other rise times longer than $1 \mathrm{~s}$. This is confirmed in Fig. 10, which shows the results of source inversions based on rupture scenarios with rise times of 2,4 and $8 \mathrm{~s}$. Scenarios are computed in the heterogeneous model A, while inversions are carried out with the homogenous background velocity model. The amplification and distortion of the slip pulse in the inversions based on data from the dense network is also observed in these cases, as found for the rise time of $1 \mathrm{~s}$ (Fig. 6), suggesting that our observations are independent of the rise time if it is longer than $1 \mathrm{~s}$. Resolving rise times shorter than $1 \mathrm{~s}$ requires a finer temporal discretization of the source and, more importantly, higher-frequency data.

\section{DISCUSSION}

With the increasing density of ground-motion data sets, the precomputed Green's function approach for source inversion becomes too computationally demanding. Standard matrix decomposition techniques are impractical on very large systems and the need arises for Krylov subspace iteration methods, like the conjugate gradient method. Particularly when the storage of the $G$ matrix is not a feasible option, adjoint approaches provide alternative means of obtaining the gradient of an objective function. As long as 1-D Green's functions represent the local Earth structure adequately, the pre-computed Green's function approach for source inversion is advantageous even for the most densely recorded earthquakes to date. However, with the improvement of crustal velocity models at regional and local scales and with the need to push earthquake source studies to higher frequencies, the demand and motivation for using 3-D Green's functions in source inversions will increase. In such cases, the adjoint approach proposed here has an edge over the pre-computed Green's functions approach, especially with increasing station density and increasing data frequency.

Generally speaking, the linear slip inversion is an underdetermined inverse problem. As such, it is subject to many biases when regularization is used. The biases are most severe when the station coverage is not regular (Zahradník \& Gallovič 2010; Gallovič \& Zahradník 2011), which is not the case of the present study. Here, regularization is implicitly achieved by stopping the CG after a fixed number of iterations (in this case fifty based on Somala et al. 2014). Better understanding of biases such as the bending of the slip pulse in Fig. 7(b) would be gained by Singular Value Decomposition analysis, for example, Gallovič \& Ampuero (2015). Unfortunately, for very dense networks such approach is impractical due to the large size of the $G$ matrix.

Underdetermined inverse problems, such as finite earthquake source inversion, tend to bias the solution towards rough models, making it hard to resolve smooth models (Das \& Suhadolc 1996; Das et al. 1996). Moreover, the homogeneous half-space model considered in our inversions is an unfavourable case in which the Green's functions from deeper sources are very similar to each other, carry redundant information, and cause a large null-space of the inverse problem. If the crustal model were more complex, providing more variable Green's functions due to reflected, refracted and scattered phases, the inversion would be better constrained.

Uncertainties of the velocity model get mapped into the model by the source inversion. This correlative noise, unlike additive noise, introduces spurious features in the retrieved model, like bending of the rupture front, bias of large slip rate values towards the surface, and widening of the rupture pulse. These issues occur in addition to the issues that appear in inversions based on known velocity models, like the loss of resolution with depth. The widening of a rupture pulse is accompanied by a reduction in slip rate amplitude. Distortion of the recovered source in the along-dip direction is manifested by an enhanced slip rate close to free surface and reduced slip rate amplitude at depth. The bending of pulses along depth can be to either side corresponding to either advanced or delayed rupture speed, depending on the properties of the medium and on the station density. The patterns of the pulse bending appears to be less susceptible to the characteristics of the medium in the coarse station network than in the dense network considered here. Irrespective of network density, the rupture velocity at shallow depth is well recovered even when data contain correlative noise. 

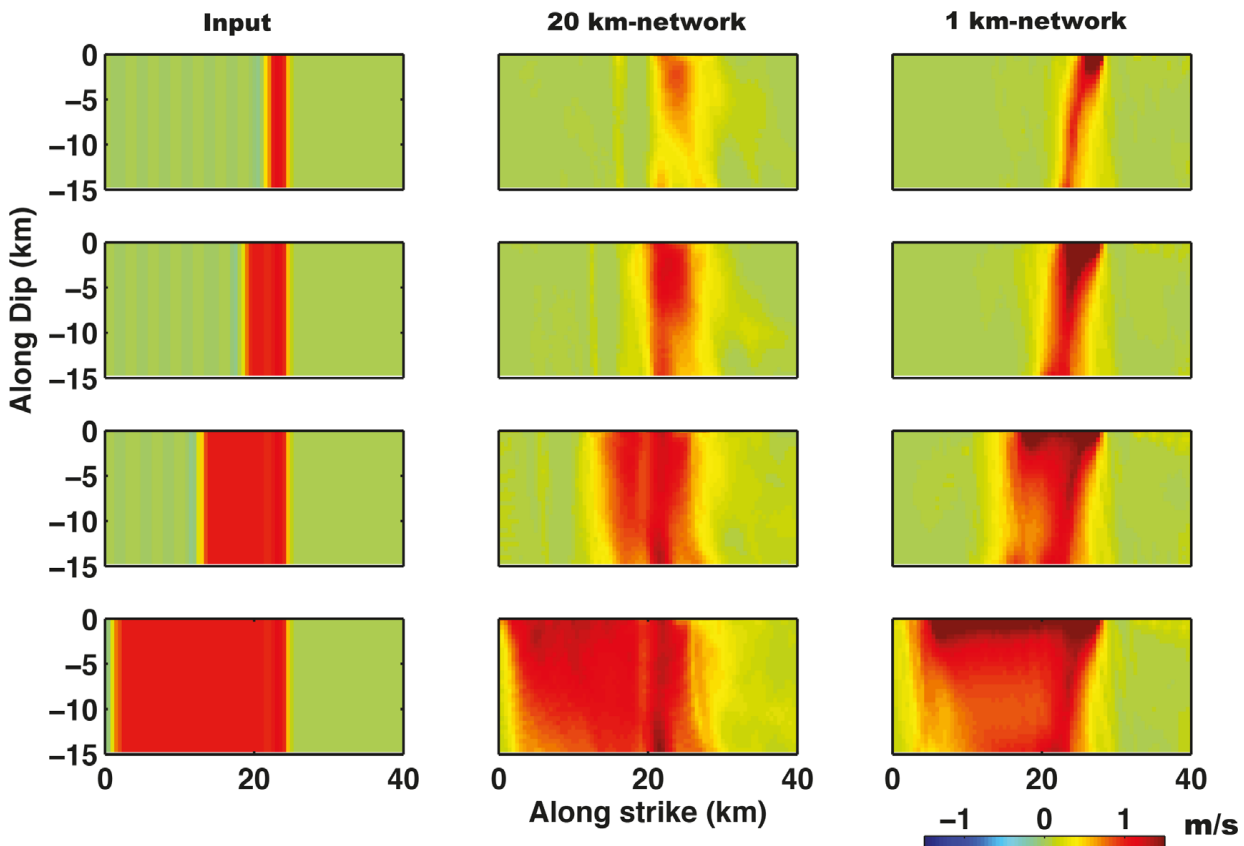

Figure 10. Effect of the rise time on source inversions. The data are generated in model A and inversion assumes the homogeneous background velocity model. The description of the three columns is the same as that given in Fig. 6 . Each row shows a representative snapshot of slip rate for rise times $1,2,4$ and $8 \mathrm{~s}$, respectively, from top to bottom. The slip pulse is distorted in the dense network irrespective of the rise time.

Constraints like regularization, positivity and smoothing can be implemented in adjoint methods, although at the expense of additional computational cost. For example, one can add to the cost function a regularization parameter multiplying the square of the gradient of the model. The resultant gradient of the cost function will have an additional term which is the regularization parameter times the model Laplacian. Similarly, Laplacian smoothing can also be incorporated in the cost function. Adding a positivity constraint deprives the inverse problem of its linearity. Linear slip inversion gives flexibility to resolve more realistic ruptures like multiple pulses and slip reversals, an idea previously identified by Somala et al. (2014) and later considered by Gallovič et al. (2015) as well as Song \& Dalguer (2017). Solving the nonlinear conjugate gradient requires a line search that involves three wave propagation simulations per iteration (Tape et al. 2007), instead of two simulations in linear case. A constraint on rake angle could also have been imposed to minimize the leakage of slip into components that had no slip in the target model, but such leakage was not a significant issue in our examples.

The choice of basis functions to represent slip rate should be based on the constraints imposed. For instance, the projection of a non-negative slip rate on high-order basis functions (linear, splines, etc.) can yield negative values (see Appendix C), which complicates the enforcement of non-negativity constraints on slip rate. Other basis functions widely used, such as wavelets (Ji et al. 2002), may also introduce such negative values; end users need to be cautious when imposing positivity constraints. A straightforward implementation of the positivity constraint for this purpose is by formulating the inversion in terms of the logarithm of slip rate as suggested by Tarantola (2005) and as performed in Gallovič et al. (2009). To avoid a number of free parameters in the inversion that is too large, the duration of the temporal basis functions in the pre-computed Green's function approach needs to be larger than the time step of the wave propagation simulations (dictated by a stability criterion).
However, it should be small enough to explain most (say 99\%) of the data, as discussed in Section 2.4 and Fig. 2.

Regularly sampled seismic networks like those considered in this study are futuristic and have not yet been deployed to record large earthquakes. The recent development of dense arrays of nodal seismic sensors, the so-called Large-N arrays, demonstrates how unaliased sampling of the wave field enables imaging seismicity at depth (Inbal et al. 2015, 2016). In our study, we set the spacing between stations and the closest distance between stations and the fault to be the same. Stations too close to the fault tend to emphasize the resolution of slip at shallow depth and farthest stations contribute little to the inversion. A non-uniform station density, decreasing with distance from the fault up to a certain extent and then increasing gradually, may be a desirable network geometry to better constrain source parameters. The optimization of a seismic network geometry for finite source inversions is an interesting and challenging topic in the experiment design.

\section{CONCLUSION}

A method for earthquake source inversion accounting for 3-D heterogeneities of the crustal velocity model is presented. The method is based on an adjoint linear formulation and it is implemented here using wave propagation simulated with a spectral element method. The adjoint approach is more computationally advantageous than the conventional approach based on pre-computed Green's functions, for dense enough sensor networks. The adjoint inversion adequately retrieves spatio-temporal details of the rupture process when the 3-D velocity structure is known. We find that typical uncertainties in crustal velocity models, represented here as a random field with $5 \mathrm{~km}$ correlation length and 5\% standard deviation, significantly degrade the quality of the source inversions based on data low-pass filtered below $1 \mathrm{~Hz}$. However, if the velocity uncertainties have a shorter correlation length of $0.5 \mathrm{~km}$ or can be reduced to a standard deviation of $1 \%$, then source inversion has an adequate 
quality. These conclusions are independent of the rise time, if it is longer than $1 \mathrm{~s}$ for the assumed $1 \mathrm{~Hz}$ upper frequency limit and velocity distributions. The method presented here is an efficient way to incorporate information about lateral heterogeneity into earthquake source inversions, and to take full advantage of the increasing availability of 3-D tomography models at regional and local scales. The method is also an essential tool to exploit future recordings of large earthquakes by very dense networks, which can enable the extension of source inversion to higher frequencies, as well as joint inversions of the source and structure, in order to advance the resolution of earthquake rupture processes.

\section{REFERENCES}

Akcelik, V. et al., 2003. High resolution forward and inverse earthquake modeling on terascale computers, in Proceedings of the 2003 ACM/IEEE conference on Supercomputing, Phoenix, AZ, p. 52, ACM.

Aki, K. \& Richards, P.G., 2002. Quantitative Seismology, University Science Books.

Asano, K. \& Iwata, T., 2009. Source rupture process of the 2004 Chuetsu, Mid-Niigata Prefecture, Japan, earthquake inferred from waveform inversion with dense strong-motion data, Bull. seism. Soc. Am., 99(1), 123-140.

Askan, A. \& Bielak, J., 2008. Full anelastic waveform tomography including model uncertainty, Bull. seism. Soc. Am., 98(6), 2975-2989.

Beresnev, I.A., 2003. Uncertainties in finite-fault slip inversions: to what extent to believe? (A critical review), Bull. seism. Soc. Am., 93(6), 24452458.

Beroza, G.C. \& Spudich, P., 1988. Linearized inversion for fault rupture behavior: application to the 1984 Morgan Hill, California, earthquake, $J$. geophys. Res., 93(B6), 6275-6296.

Bouchon, M., Toksöz, M.N., Karabulut, H., Bouin, M.-P., Dietrich, M., Aktar, M. \& Edie, M., 2002. Space and time evolution of rupture and faulting during the 1999 Izmit (Turkey) earthquake, Bull. seism. Soc. Am., 92(1), 256-266.

Chi, W.-C., Dreger, D. \& Kaverina, A., 2001. Finite-source modeling of the 1999 Taiwan (Chi-Chi) earthquake derived from a dense strong-motion network, Bull. seism. Soc. Am., 91(5), 1144-1157.

Claerbout, J.F. \& Fomel, S., 2008. Image Estimation by Example: Geophysical Soundings Image Construction: Multidimensional Autoregression, Stanford University.

Clayton, R.W. et al., 2012. Community seismic network, Ann. Geophys., 54(6), doi:10.4401/ag-5269.

Das, S. \& Suhadolc, P., 1996. On the inverse problem for earthquake rupture: the Haskell-type source model, J. geophys. Res., 101(B3), 5725-5738.

Das, S., Suhadolc, P. \& Kostrov, B.V., 1996. Realistic inversions to obtain gross properties of the earthquake faulting process, Tectonophysics, 261(1), 165-177.

Delouis, B., Giardini, D., Lundgren, P. \& Salichon, J., 2002. Joint inversion of InSAR, GPS, teleseismic, and strong-motion data for the spatial and temporal distribution of earthquake slip: application to the 1999 Izmit mainshock, Bull. seism. Soc. Am., 92(1), 278-299.

Duputel, Z. et al., 2015. The iquique earthquake sequence of april 2014: Bayesian modeling accounting for prediction uncertainty, Geophys. Res. Lett., 42(19), 7949-7957.

Fichtner, A., Kennett, B.L., Igel, H. \& Bunge, H.-P., 2010. Full waveform tomography for radially anisotropic structure: new insights into present and past states of the Australasian upper mantle, Earth planet. Sci. Lett., 290(3-4), 270-280.

Frankel, A. \& Clayton, R.W., 1986. Finite difference simulations of seismic scattering: implications for the propagation of short-period seismic waves in the crust and models of crustal heterogeneity, J. geophys. Res., 91(B6), 6465-6489.

Gabriel, A.-A., Ampuero, J.-P., Dalguer, L.A. \& Mai, P.M., 2012. The transition of dynamic rupture styles in elastic media under velocity-weakening friction, J. geophys. Res., 117(B9), doi:10.1029/2012JB009468.
Gallovič, F. \& Ampuero, J.-P., 2015. A new strategy to compare inverted rupture models exploiting the eigenstructure of the inverse problem, Seismol. Res. Lett., 86(6), 1679-1689.

Gallovič, F. \& Zahradník, J., 2011. Toward understanding slip inversion uncertainty and artifacts: 2. Singular value analysis, J. geophys. Res., 116(B2), doi:10.1029/2010JB007814.

Gallovič, F. \& Zahradník, J., 2012. Complexity of the mw 6.3 2009 L'Aquila (central Italy) earthquake: 1. Multiple finite-extent source inversion, $J$. geophys. Res., 117(B4), doi:10.1029/2011JB008729.

Gallovič, F., Zahradník, J., Kř́żová, D., Plicka, V., Sokos, E., Serpetsidaki, A. $\&$ Tselentis, G.-A., 2009. From earthquake centroid to spatial-temporal rupture evolution: $M_{\mathrm{W}} 6.3$ Movri Mountain earthquake, June 8, 2008, Greece, Geophys. Res. Lett., 36(21), doi:10.1029/2009GL040283.

Gallovič, F., Imperatori, W. \& Mai, P.M., 2015. Effects of three-dimensional crustal structure and smoothing constraint on earthquake slip inversions: case study of the $M_{\mathrm{w}} 6.32009$ L'Aquila earthquake, J. geophys. Res., 120(1), 428-449.

Galvez, P., Dalguer, L.A., Ampuero, J.-P. \& Giardini, D., 2016. Rupture reactivation during the $2011 M_{\mathrm{W}} 9.0$ Tohoku earthquake: dynamic rupture and ground-motion simulations, Bull. seism. Soc. Am., 106(3), 819-831.

Graves, R.W. \& Wald, D.J., 2001. Resolution analysis of finite fault source inversion using one- and three-dimensional Green's functions: 1. Strong motions, J. geophys. Res., 106(B5), 8745-8766.

Gülen, L., Pinar, A., Kalafat, D., Özel, N., Horasan, G., Yilmazer, M. \& Işikara, A.M., 2002. Surface fault breaks, aftershock distribution, and rupture process of the 17 August 1999 Izmit, Turkey, earthquake, Bull. seism. Soc. Am., 92(1), 230-244.

Hallo, M. \& Gallovič, F., 2016. Fast and cheap approximation of green function uncertainty for waveform-based earthquake source inversions, Geophys. Suppl. Mon. Not. R. astr. Soc., 207(2), 1012-1029.

Hartzell, S., Harmsen, S. \& Frankel, A., 2010. Effects of 3D random correlated velocity perturbations on predicted ground motions, Bull. seism. Soc. Am., 100(4), 1415-1426.

Hartzell, S.H. \& Heaton, T.H., 1983. Inversion of strong ground motion and teleseismic waveform data for the fault rupture history of the 1979 Imperial Valley, California, earthquake, Bull. seism. Soc. Am., 73(6A), 1553-1583.

Hestenes, M. \& Stiefel, E., 1952. Methods of conjugate gradients for solving linear systems, J. Res. Natl. Bur. Stand., 49(6), 409-436.

Hjörleifsdóttir, V., Simons, M. \& Tromp, J., 2007, Earthquake source characterization using 3D numerical modeling, $P h D$ thesis, California Institute of Technology, Pasadena, CA.

Ide, S., Beroza, G.C. \& McGuire, J.J., 2005. Imaging earthquake source complexity, in Geophysical Monograph Series, Vol. 157, pp. 117-135, eds Levander, A. \& Nolet, G., American Geophysical Union.

Inbal, A., Clayton, R.W. \& Ampuero, J.-P., 2015. Imaging widespread seismicity at midlower crustal depths beneath Long Beach, CA, with a dense seismic array: evidence for a depth-dependent earthquake size distribution, Geophys. Res. Lett., 42(15), 6314-6323.

Inbal, A., Ampuero, J.P. \& Clayton, R.W., 2016. Localized seismic deformation in the upper mantle revealed by dense seismic arrays, Science, 354(6308), 88-92.

Ji, C., Wald, D.J. \& Helmberger, D.V., 2002. Source description of the 1999 Hector Mine, California, earthquake, Part I: wavelet domain inversion theory and resolution analysis, Bull. seism. Soc. Am., 92(4), 1192-1207.

Kawakatsu, H. \& Montagner, J.-P., 2008. Time-reversal seismic-source imaging and moment-tensor inversion, Geophys. J. Int., 175(2), 686-688.

Kim, Y., Liu, Q. \& Tromp, J., 2011. Adjoint centroid-moment tensor inversions, J. geophys. Int., 186(1), 264-278.

Komatitsch, D., Erlebacher, G., Göddeke, D. \& Michéa, D., 2010. Highorder finite-element seismic wave propagation modeling with MPI on a large GPU cluster, J. Comput. Phys., 229(20), 7692-7714.

Kremers, S., Fichtner, A., Brietzke, G.B., Igel, H., Larmat, C., Huang, L. \& Käser, M., 2011. Exploring the potentials and limitations of the timereversal imaging of finite seismic sources, Solid Earth, 2(1), 95-105.

Lee, S.-J., 2012. Rupture process of the 2011 Tohoku-Oki earthquake based upon joint source inversion of teleseismic and GPS data, Terr. Atmos. Ocean. Sci., doi:10.3319/TAO.2011.07.11.01(T). 
Lee, S.-J., Huang, B.-S., Ando, M., Chiu, H.-C. \& Wang, J.-H., 2011. Evidence of large scale repeating slip during the 2011 Tohoku-Oki earthquake, Geophys. Res. Lett., 38(19), L19306.

Lee, W.H.K., Shin, T.C., Kuo, K.W., Chen, K.C. \& Wu, C.F., 2001. CWB free-field strong-motion data from the 21 September Chi-Chi, Taiwan, earthquake, Bull. seism. Soc. Am., 91(5), 1370-1376.

Li, X., Cormier, V.F. \& Toksöz, M.N., 2002. Complex source process of the 17 August 1999 Izmit, Turkey, earthquake, Bull. seism. Soc. Am., 92(1), 267-277.

Liu, P. \& Archuleta, R.J., 2004. A new nonlinear finite fault inversion with three-dimensional Green's functions: application to the 1989 Loma Prieta, California, earthquake, J. geophys. Res., 109(B2), doi:10.1029/2003JB002625.

Liu, P., Custódio, S. \& Archuleta, R.J., 2006. Kinematic inversion of the 2004 M 6.0 Parkfield earthquake including an approximation to site effects, Bull. seism. Soc. Am., 96(4B), S143-S158.

Liu, Q. \& Tromp, J., 2006. Finite-frequency kernels based on adjoint methods, Bull. seism. Soc. Am., 96(6), 2383-2397.

Liu, Q. \& Tromp, J., 2008. Finite-frequency sensitivity kernels for global seismic wave propagation based upon adjoint methods, J. geophys. Int., 174(1), 265-286.

Ma, K.-F., Mori, J., Lee, S.-J. \& Yu, S.B., 2001. Spatial and temporal distribution of slip for the 1999 Chi-Chi, Taiwan, earthquake, Bull. seism. Soc. Am., 91(5), 1069-1087.

Mai, P.M. et al., 2016. The earthquake-source inversion validation (SIV) project, Seismol. Res. Lett., 87, 690-708.

Michel, R., Ampuero, J., Avouac, J., Lapusta, N., Leprince, S., Redding, D. \& Somala, S.N., 2013. A geostationary optical seismometer, proof of concept, IEEE Trans. Geosci. Remote Sens., 51(1), 695-703.

Okada, Y., 2013. Recent progress of seismic observation networks in japan, Journal of Physics: Conference Series, 433(1), 012039.

Olson, A.H. \& Anderson, J.G., 1988. Implications of frequency-domain inversion of earthquake ground motions for resolving the space-time dependence of slip on an extended fault, Geophys. J., 94(3), 443-455.

Olson, A.H. \& Apsel, R.J., 1982. Finite faults and inverse theory with applications to the 1979 Imperial Valley earthquake, Bull. seism. Soc. Am., 72(6A), 1969-2001.

Polak, E. \& Ribière, G., 1969. Note sur la convergence de directions conjugées, Rev. Française Informat, Rech. Oper., 3e année, 16, 35-43.

Sekiguchi, H. \& Iwata, T., 2002. Rupture process of the 1999 Kocaeli, Turkey, earthquake estimated from strong-motion waveforms, Bull. seism. Soc. Am., 92(1), 300-311.

Sekiguchi, H., Irikura, K. \& Iwata, T., 2000. Fault geometry at the rupture termination of the 1995 Hyogo-ken Nanbu earthquake, Bull. seism. Soc. Am., 90(1), 117-133.

Shao, G., Ji, C. \& Hauksson, E., 2012. Rupture process and energy budget of the 29 July $2008 \mathrm{Mw}$ 5.4 Chino Hills, California, earthquake, J. geophys. Res., 117(B7), doi:10.1029/2011JB008856.

Somala, S.N., Ampuero, J.-P. \& Lapusta, N., 2014. Resolution of rise time in earthquake slip inversions: effect of station spacing and rupture velocity, Bull. seism. Soc. Am., 104(6), 2717-2734.

Song, S.G. \& Dalguer, L.A., 2017. Synthetic source inversion tests with the full complexity of earthquake source processes, including both supershear rupture and slip reactivation, Pure appl. Geophys., 174(9), 3393-3418.

Tape, C., Liu, Q. \& Tromp, J., 2007. Finite-frequency tomography using adjoint methods-methodology and examples using membrane surface waves, Geophys. J. Int., 168(3), 1105-1129.

Tape, C., Liu, Q., Maggi, A. \& Tromp, J., 2009. Adjoint tomography of the southern California crust, Science, 325(5943), 988-992.

Tape, C., Liu, Q., Maggi, A. \& Tromp, J., 2010. Seismic tomography of the southern California crust based on spectral-element and adjoint methods, Geophys. J. Int., 180(1), 433-462.

Tarantola, A., 1984. Inversion of seismic reflection data in the acoustic approximation, Geophysics, 49(8), 1259-1266.

Tarantola, A., 2005. Inverse Problem Theory and Methods for Model Parameter Estimation, SIAM.
Tromp, J., Tape, C. \& Liu, Q., 2005. Seismic tomography, adjoint methods, time reversal and banana-doughnut kernels, Geophys. J. Int., 160(1), 195216

Yagi, Y. \& Fukahata, Y., 2008. Importance of covariance components in inversion analyses of densely sampled observed data: an application to waveform data inversion for seismic source processes, Geophys. J. Int., 175(1), 215-221.

Yu, S.-B. et al., 2001. Preseismic deformation and coseismic displacements associated with the 1999 Chi-Chi, Taiwan, earthquake, Bull. seism. Soc. Am., 91(5), 995-1012.

Zahradník, J. \& Gallovič, F., 2010. Toward understanding slip inversion uncertainty and artifacts, J. geophys. Res., 115(B9), doi:10.1029/2010JB007414.

Zeng, Y. \& Chen, C.-H., 2001. Fault rupture process of the 20 September 1999 Chi-Chi, Taiwan, earthquake, Bull. seism. Soc. Am., 91(5), 10881098.

\section{APPENDIX A: ESTIMATES OF COMPUTATIONAL COST OF PRE-COMPUTED GREEN'S FUNCTION AND ADJOINT METHODS}

Our adjoint method requires two simulations per iteration of the CG algorithm, one forward and one adjoint simulation. For source inversions incorporating 3-D crustal velocity models, both simulations are done with a 3-D wave propagation code, here SPECFEM3D. The total computational cost, $\Gamma_{\text {adj }}$, of an inversion with the adjoint approach is

$\Gamma_{\text {adj }}=2 N_{\text {iter }} C_{\text {SEM }}$

where $N_{\text {iter }}$ is the number of CG iterations and $C_{S E M}$ is the computational cost of a single 3-D SEM wave propagation simulation. The latter is quantified here by the number of floating point multiplications, which we evaluated by inspection of the SPECFEM3D code, mainly in the routines corresponding to the elastic force computation and the Newmark time stepping:

$C_{\mathrm{SEM}}=N_{t}^{\mathrm{SEM}} N_{e} \times 9\left(2 N_{\mathrm{GLL}}+9\right) N_{\mathrm{GLL}}^{3}$,

where $N_{t}^{\text {SEM }}$ is the number of time steps of the SEM simulation and $N_{e}$ the number of spectral elements.

The total cost of the GF approach, $\Gamma_{\mathrm{GF}}$, comprises the cost per $\mathrm{CG}$ iteration plus the overhead cost of pre-computing the Green's functions, $\Gamma_{\mathrm{GF}}^{\mathrm{oh}}$ :

$\Gamma_{\mathrm{GF}}=2 N_{\mathrm{iter}} C_{\mathrm{conv}}+\Gamma_{\mathrm{GF}}^{\mathrm{oh}}$.

The number of CG iterations is the same as in the adjoint approach, $N_{\text {iter }}$. The cost per iteration is dominated by the cost, $2 C_{\text {conv }}$, of convolutions between the Green's functions and the slip rate timeseries in step 6 of the CG algorithm, and cross-correlations between Green's functions and residual ground velocity time-series in step 9. We assume the convolutions and cross-correlations are done employing the fast Fourier transform (FFT). This approach is more efficient than direct time-domain evaluation because the seismograms and the slip-rate functions have comparable durations, due to our unrestricted parametrization of the source. Each of these operations involves essentially two FFTs per data channel $\left(N_{\text {sta }}\right.$ stations with three components of ground motion) and per model channel ( $N_{\text {fault }}$ fault cells with two components of slip rate). The time-series contain $N_{t}^{\text {data }}$ samples and the FFTs include zero-padding to twice this length. The number of floating point operations per FFT is $O(10) 2 N_{t}^{\text {data }} \log _{2}\left(2 N_{t}^{\text {data }}\right)$. Hence,

$C_{\text {conv }}=2 N_{\text {fault }} \times 3 N_{\text {sta }} \times O(10) 4 N_{t}^{\text {data }} \log _{2}\left(2 N_{t}^{\text {data }}\right)$. 
The overhead involves $2 N_{\text {fault }}$ SPECFEM3D wave propagation simulations. If reciprocity is exploited, $3 N_{\text {sta }}$ simulations are needed. Hence,

$\Gamma_{\mathrm{GF}}^{\mathrm{oh}}=\min \left(2 N_{\text {fault }}, 3 N_{\mathrm{sta}}\right) \times C_{\mathrm{SEM}}$.

Eqs (A1)-(A5) are combined to obtain the ratio of computational cost between the GF and adjoint approaches

$\frac{\Gamma_{\mathrm{GF}}}{\Gamma_{\mathrm{adj}}}=\frac{C_{\mathrm{conv}}}{C_{\mathrm{SEM}}}+\frac{\Gamma_{\mathrm{GF}}^{\mathrm{oh}}}{\Gamma_{\mathrm{adj}}}$

with

$\frac{C_{\mathrm{conv}}}{C_{\mathrm{SEM}}}=O(10) \frac{24}{9} \frac{N_{\mathrm{fault}} N_{\mathrm{sta}} N_{t}^{\text {data }} \log _{2}\left(2 N_{t}^{\text {data }}\right)}{N_{e} N_{t}^{\mathrm{SEM}} N_{\mathrm{GLL}}^{3}\left(2 N_{\mathrm{GLL}}+9\right)}$

and

$\frac{\Gamma_{\mathrm{GF}}^{\mathrm{oh}}}{\Gamma_{\mathrm{adj}}}=\frac{\min \left(N_{\mathrm{fault}}, 3 / 2 N_{\mathrm{sta}}\right)}{N_{\mathrm{iter}}}$.

To develop the order-of-magnitude estimates given in eqs (33) and (34) for all the terms involved in eqs (A6)-(A8), we adopt the following considerations and assumptions:

(1) In a spectral element simulation with polynomial order $N_{\mathrm{GLL}}-1 \approx 4$ and minimum resolution wavelength $\lambda$, the spectral element size is $\approx \lambda$ and the fault cell size is $\Delta x_{m} \approx \lambda /\left(N_{\mathrm{GLL}}-1\right)$.

(2) The number of fault cells on a rupture area of length $L$ and width $W$ is $N_{\text {fault }}=L W / \Delta x_{m}^{2}$.

(3) The number of time steps $N_{t}^{\text {data }}$ for the GF approach is the total duration of the seismograms, $T$, divided by the source time step, $\Delta t_{m} \approx 0.1 / f_{c}$ where $f_{c}=c_{S} / \lambda$ is the upper cut-off frequency of the data (Section 2.4): $N_{t}^{\text {data }} \approx 10 T \lambda / c_{S}$. For $T f_{c}=10^{2} \sim 10^{4}$, a range covering most practical situations, $\log _{2}\left(2 N_{t}^{\text {data }}\right)=O(10)$.

(4) The number of spectral elements, $N_{e}$, is the domain volume (a semi-sphere of radius a couple of fault lengths) divided by the volume of a spectral element: $N_{e}=\frac{2}{3} \pi(2 L / \lambda)^{3}$.

(5) The SEM time step is $\Delta t_{\mathrm{SEM}} \approx 2 \lambda /\left(c_{p} N_{\mathrm{GLL}}^{2}\right)$. The total number of SEM time steps is $N_{t}^{\mathrm{SEM}}=T / \Delta t_{\mathrm{SEM}} \approx \frac{1}{2} c_{p} N_{\mathrm{GLL}}^{2} T / \lambda$.

(6) The number of stations with spacing $\Delta x_{s}$ on a disk of radius equal to a couple of fault lengths is $N_{\text {sta }}=\pi\left(2 L / \Delta x_{s}\right)^{2}$.

(7) $c_{P} / c_{S}=\sqrt{3}$

(8) In our experience so far, $N_{\text {iter }}=O(100)$ or less.

\section{APPENDIX B: WAVEFORM FITS}

The three-component target velocities together with synthetic velocity waveforms based on coarse and dense networks, in the frequency band of inversion, at five stations common to both networks (Fig. B1) are shown in Figs B2-B5, for all four inversions performed in this study. Fig. B2 corresponds to the only 3-D inversion where data are generated using heterogeneous velocity model and inversion also uses heterogeneous velocity model. Figs B3-B5 correspond to data generated using 3-D heterogeneous velocity models $\mathrm{A}, \mathrm{B}$ and $\mathrm{C}$ respectively but inversion uses the background velocity model. Fig. B2 conveys that the synthetics from the inverted model for coarse and dense networks are almost overlapping with the raw data, within the frequency band of inversion, for the inversion where the heterogeneity is well known. Even in cases where heterogeneity is not well known (Figs B3-B5), if the unknown heterogeneity is within certain limits, the waveform fit appears to be near perfect.

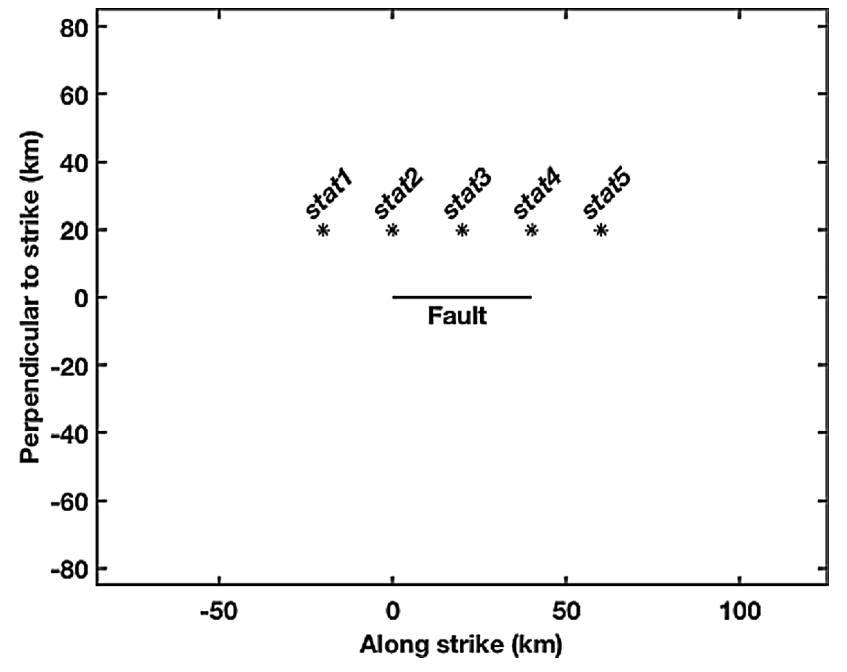

Figure B1. Map view of stations for which waveform comparison is shown in Figs B2-B5.

\section{APPENDIX C: HIGHER ORDER REPRESENTATIONS DO NOT PRESERVE POS I T IVIT Y}

Through an example we show that the representation of a nonnegative function by linear basis functions might introduce negative values. Consider a representation $m(t)$ of a continuous slip rate function $v(t)$ in terms of orthonormal linear temporal basis functions $b_{q}(t)$ :

$v(t) \approx m(t)=\sum_{p=0}^{N_{t}} m_{p} b_{p}(t)$

where

$b_{q}(t)= \begin{cases}\frac{t-t_{q-1}}{t_{q}-t_{q-1}} & \text { if } t \in\left[t_{q-1}, t_{q}\right] \\ \frac{t_{q+1}-t}{t_{q+1}-t_{q}} & \text { if } t \in\left[t_{q}, t_{q+1}\right] . \\ 0 & \text { otherwise }\end{cases}$

The coefficients $m_{p}$ are found by solving

$\int_{0}^{T}(m(t)-v(t)) b_{p}(t) \mathrm{d} t=0 \quad \forall p \in\left\{0, \ldots, N_{t}\right\}$.

This leads to the following algebraic problem:

$\mathbf{B m}=\tilde{\mathbf{v}}$

where $\mathbf{B}$ is a tridiagonal matrix with components

$B_{p q}=\int_{0}^{T} b_{p}(t) b_{q}(t) \mathrm{d} t$

and $\tilde{\mathbf{v}}$ is a vector with components

$\tilde{v}_{p}=\int_{0}^{T} v(t) b_{p}(t) \mathrm{d} t$.

Consider a continuous slip rate function $v(t)$ discretized by four linear basis functions with unit width. Consider the following nonnegative vector of projected slip rates

$\tilde{\mathbf{v}}=\left[\begin{array}{l}2 \\ 1 \\ 4 \\ 3\end{array}\right]$. 

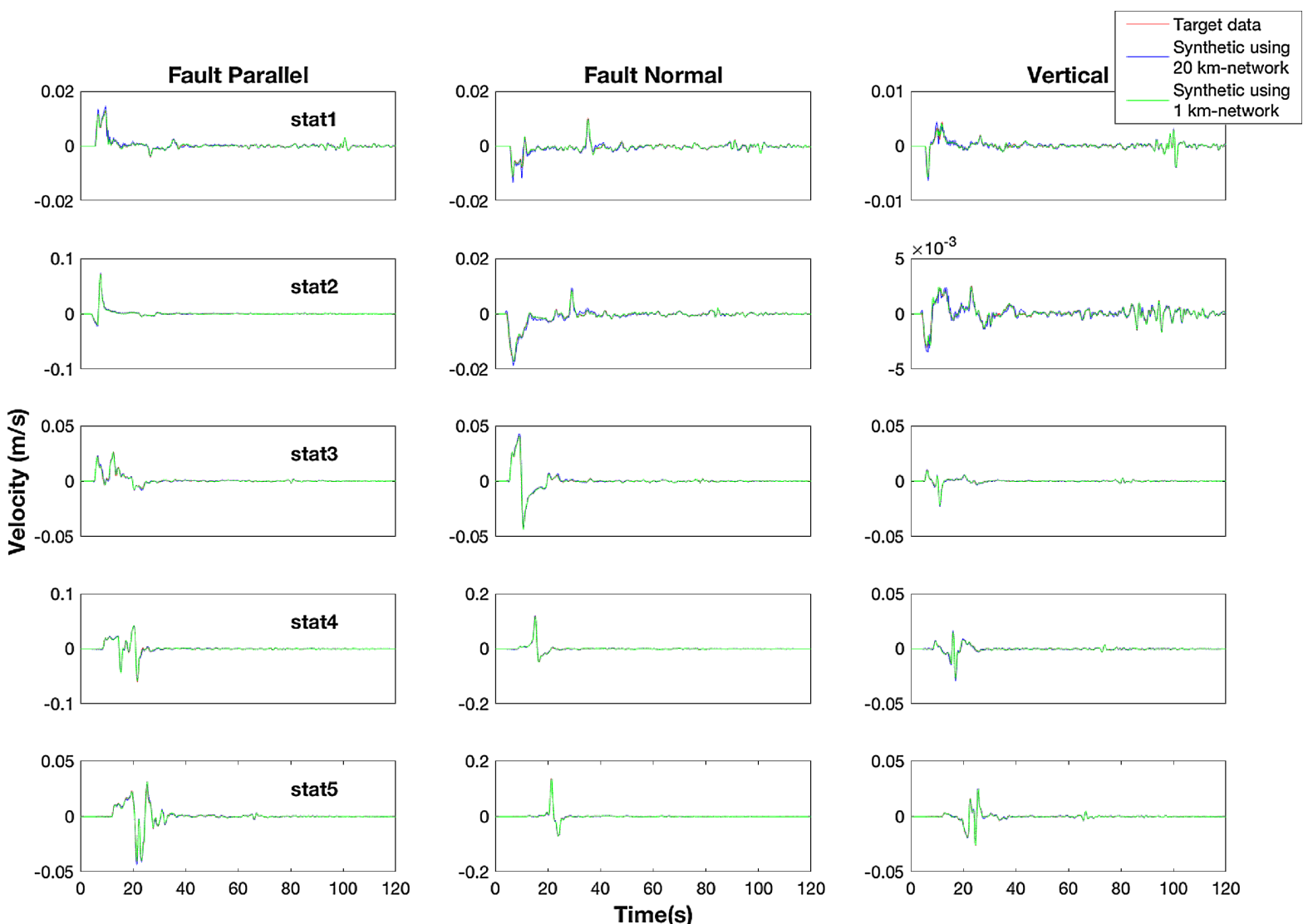

Figure B2. Data are generated using heterogeneous velocity model A. Inversion also uses the 3-D heterogeneous velocity model A.
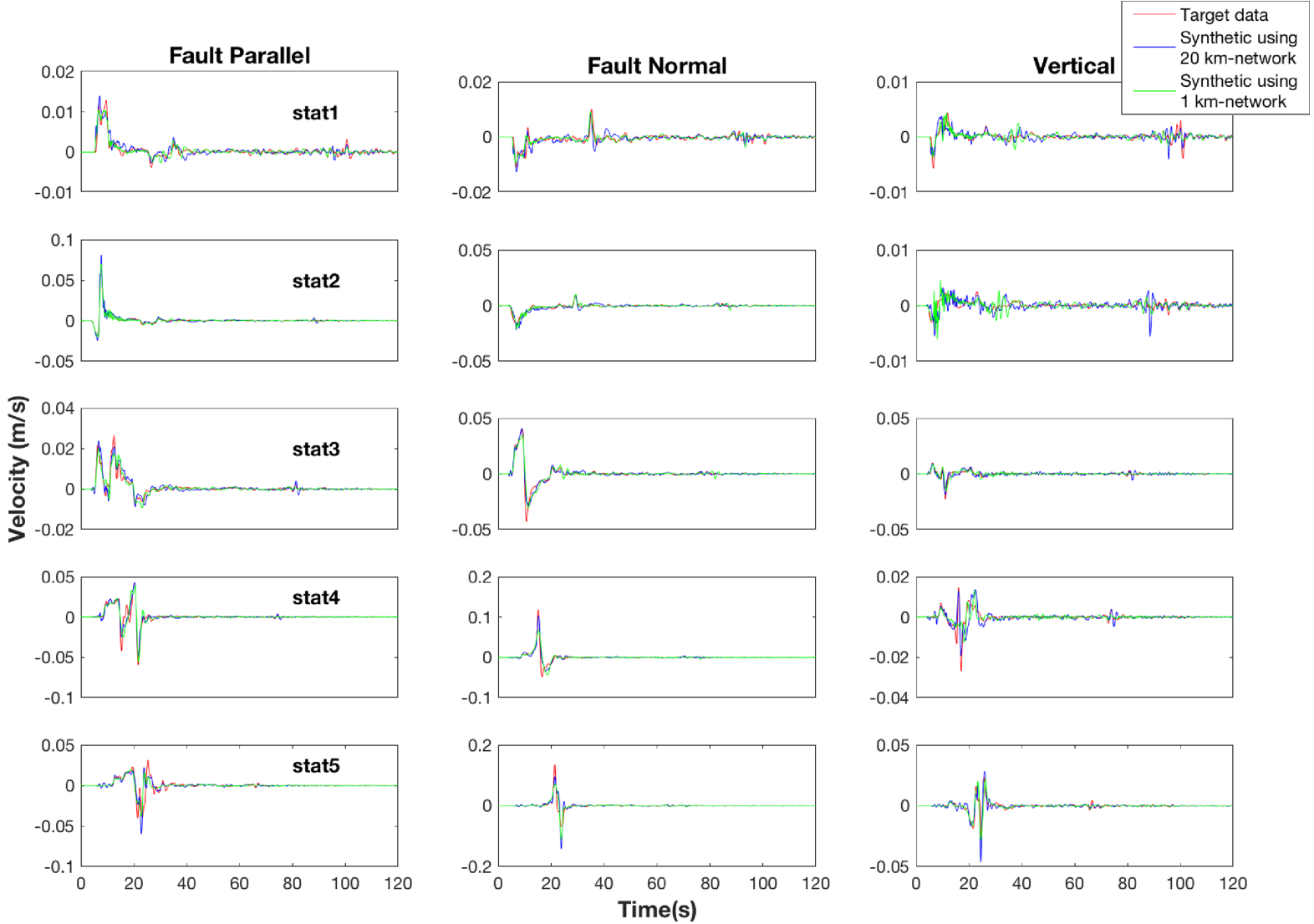

Figure B3. Data are generated using heterogeneous velocity model A. Inversion uses background velocity model. 

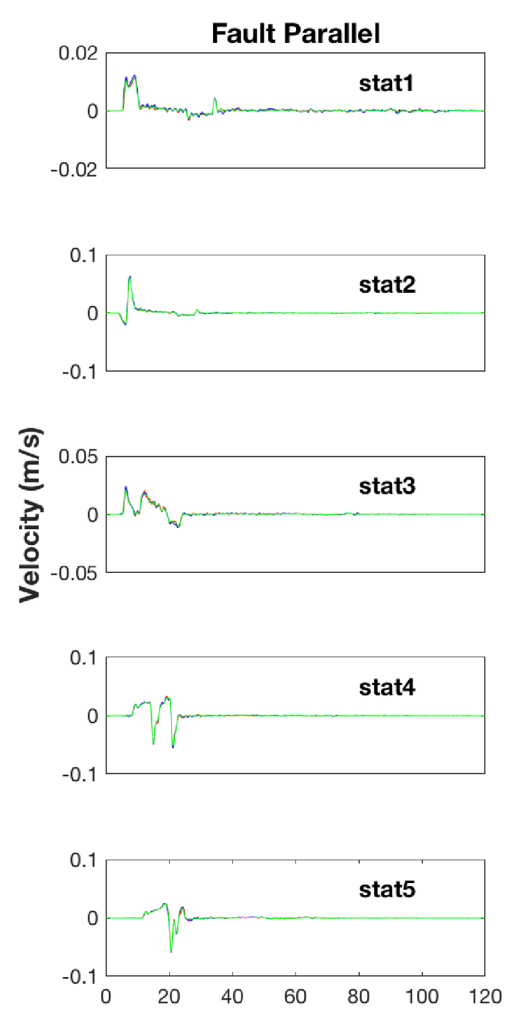
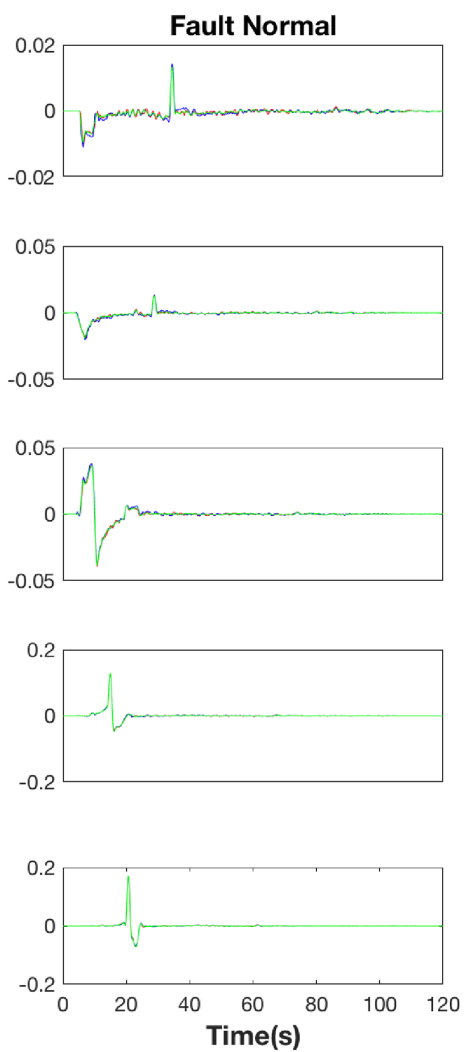
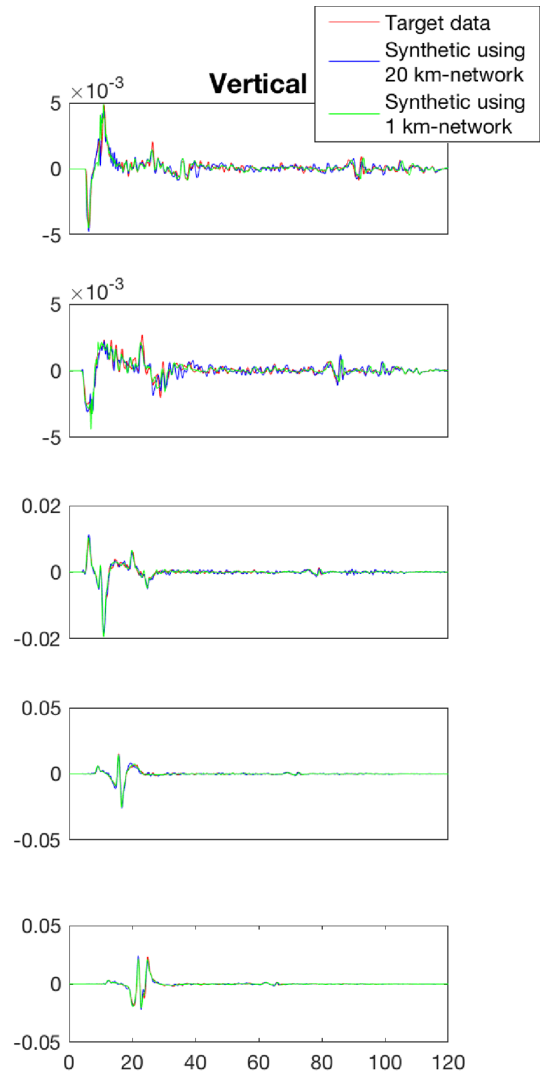

Figure B4. Data is generated using heterogeneous velocity model B. Inversion uses background velocity model.

Solving eq. (C4) with

$$
\mathbf{B}=\frac{1}{3}\left[\begin{array}{cccc}
1 & 0.5 & 0 & 0 \\
0.5 & 2 & 0.5 & 0 \\
0 & 0.5 & 2 & 0.5 \\
0 & 0 & 0.5 & 1
\end{array}\right]
$$

we obtain the model coefficients

$\mathbf{m}=\frac{1}{3}\left[\begin{array}{c}20 \\ -4 \\ 14 \\ 20\end{array}\right]$.
A negative value in the model coefficients appears even though all the projected values are non-negative. 

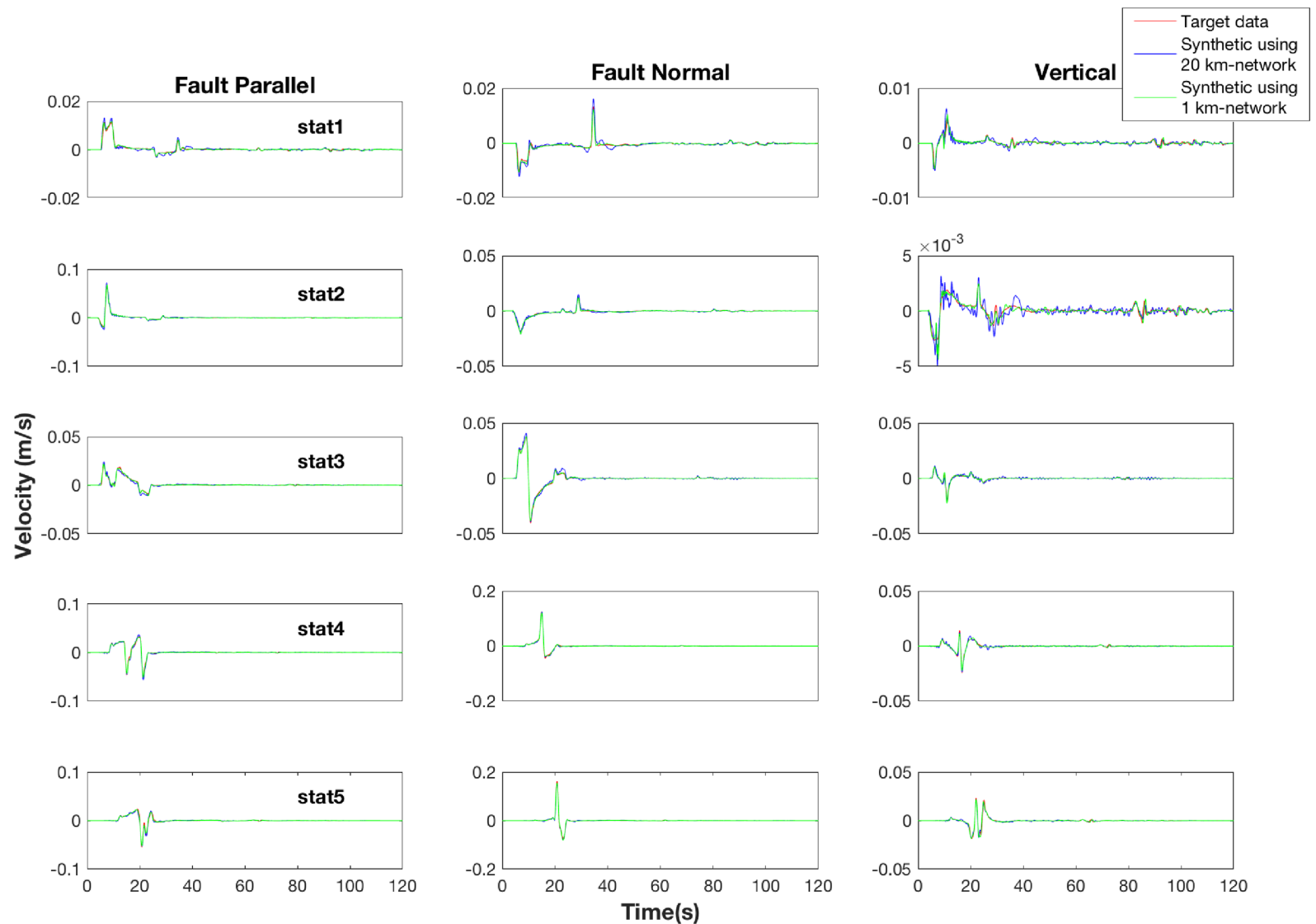

Figure B5. Data are generated using heterogeneous velocity model C. Inversion uses background velocity model. 